\title{
An expeditious synthesis of tailed tren-capped porphyrins
}

\author{
Pascale Even, Christian Ruzié, David Ricard, and Bernard Boitrel* \\ Université de Rennes1. Institut de Chimie. UMR CNRS 6509. \\ 35042 Rennes Cedex. France \\ Bernard.Boitrel@univ-rennes1.fr
}

\section{(Supplementary Material)}

\section{Materials and Methods.}

All reactions were performed under argon and were magnetically stirred. Commercially available reagents were used without further purification unless otherwise stated. Solvents were distilled over appropriate drying agent prior to use : $\mathrm{CH}_{2} \mathrm{Cl}_{2}$, triethylamine, pyridine and pentane over $\mathrm{CaH}_{2}$; THF and toluene over potassium benzophenone ketyl. Solvents used in an inert atmosphere dry box; $\left[\mathrm{O}_{2}\right]<1 \mathrm{ppm}$ ) were freshly distilled and deoxygenated by three "freeze pump" cycles. All reactions were monitored by TLC with Merck precoated aluminium foil sheets (silica gel 60 with fluorescent indicator $U_{254}$ ). Column chromatography was carried out using silicagel from Merck (TLC-Kieselgel 60H, $15 \mu \mathrm{m}$ ). ${ }^{1} \mathrm{H}$ and ${ }^{13} \mathrm{C}$ NMR spectra were recorded using Bruker Avance $500 \mathrm{dpx}$ operating at $500 \mathrm{MHz}$ and $125 \mathrm{MHz}$ or Bruker Avance $300 \mathrm{dpx}$ operating at $300 \mathrm{MHz}$ and $75 \mathrm{MHz}$ in the solvent specified and referenced to the residual proton signals. High resolution mass spectra were recorded on a Micromass MS/MS ZABSpec TOF in ESI positif mode at the CRMPO (Centre Régional de Mesure Physique de l'Ouest - Rennes, FRANCE). Liquid UV-visible spectra were recorded on a UVIKON XL spectrometer from Biotech.

\section{Synthesis and Characterization.}

\section{meso- $\alpha-5,10,15-\beta-20$-tetrakis-(2-amino)phenyl-porphyrin (TAPP $\alpha \alpha \alpha \beta)$}

The four atropisomers of the meso-5,10,15,20-tetrakis-(2-amino)phenyl-porphyrin or TAPP were separated on a silica gel column chromatography with methylene chloride. The atropisomer $\alpha \alpha \alpha \beta$ of the TAPP was eluted with $1 \% \mathrm{MeOH} / \mathrm{CH}_{2} \mathrm{Cl}_{2}$ and evaporated under vacuo without heating to avoid atropisomerisation. The mixture of remaining atropisomers was refluxed in toluene overnight to obtain a new statistic mixture and evaporated under vacuo. The atropisomer $\alpha \alpha \alpha \beta$ was obtained as described above in $50 \%$ yield.

${ }^{1} \mathrm{H} \mathrm{NMR}\left(500 \mathrm{MHz}, \mathrm{CDCl}_{3}, 323 \mathrm{~K}\right): \delta 8.93$ (brs, 8H), $7.90(\mathrm{dd}, 2 \mathrm{H}, \mathrm{Jo}=7.5 \mathrm{~Hz}, \mathrm{Jm}=1.5 \mathrm{~Hz})$, $7.86(\mathrm{t}, 2 \mathrm{H}, \mathrm{Jo}=7.0 \mathrm{~Hz}, \mathrm{Jm}=1.5 \mathrm{~Hz}), 7.62(\mathrm{t}, 4 \mathrm{H}, \mathrm{J}=8 \mathrm{~Hz}), 7.21-7.17(\mathrm{~m}, 4 \mathrm{H}), 7.15-7.13$ (m, 4H), 3.56 (brs, 8H), 2.65 (s, 2H). 


\section{meso- $\alpha-5,10,15-\beta$-20-tetrakis-(2-acryloylamino)phenyl-porphyrin $\left(1 \mathrm{H}_{2}\right)$}

In a $1 \mathrm{~L}$ round bottom flask under argon TAPP $\alpha \alpha \alpha \beta(1 \mathrm{~g}, 1.48 \mathrm{mmol})$ was dissolved in 500 $\mathrm{mL}$ of freshly distilled THF with triethylamine. Then, acryloyl chloride dissolved in $30 \mathrm{~mL}$ of THF was added dropwise at $-50^{\circ} \mathrm{C}$. The solution was stirred for 10 minutes and then, warmed to room temperature and evaporated to dryness under reduced pressure. The resulting powder was dissolved in $\mathrm{CH}_{2} \mathrm{Cl}_{2}$ and loaded on a silica gel chromatography column. The desired product eluted with $10 \%$ acetone $/ \mathrm{CH}_{2} \mathrm{Cl}_{2}$ was obtained in $50 \%$ yield $(660 \mathrm{mg})$.

${ }^{1} \mathrm{H}$ NMR $\left(500 \mathrm{MHz}, \mathrm{CDCl}_{3}, 323 \mathrm{~K}\right): \delta 8.90(\mathrm{~s}, 8 \mathrm{H}), 8.81(\mathrm{~m}, 4 \mathrm{H}), 8.02(\mathrm{~m}, 4 \mathrm{H}), 7.91(\mathrm{td}, 4 \mathrm{H}$, $\mathrm{Jo}=8.0 \mathrm{~Hz}, \mathrm{Jm}=1.3 \mathrm{~Hz}), 7.59(\mathrm{td}, \mathrm{Jo}=7.5 \mathrm{~Hz}, \mathrm{Jm}=1.4 \mathrm{~Hz}, 4 \mathrm{H}), 6.97(\mathrm{~s}, 2 \mathrm{H}), 6.96(\mathrm{~s}, 1 \mathrm{H})$, $6.89(\mathrm{~s}, 1 \mathrm{H}), 5.85-5.66(\mathrm{~m}, 4 \mathrm{H}), 5.19-5.00(\mathrm{~m}, 8 \mathrm{H}),-2.52(\mathrm{~s}, 2 \mathrm{H})$.

${ }^{13} \mathrm{C}$ NMR $\left(125 \mathrm{MHz}\right.$, pyridine- $\left.d_{5}, 323 \mathrm{~K}\right): \delta$ 163.6, 135.4, 135.0, 132.3, 131.2, 131.0, 130.5, $127.4,127.2,123.8,122.3,122.1$.

$\lambda_{\text {abs }}\left(\mathrm{CH}_{2} \mathrm{Cl}_{2}\right)\left(\varepsilon, \mathrm{mM}^{-1} \mathrm{~cm}^{-1}\right) 420$ (253.9), 513 (14.7), 546 (3.9), 587 (4.8), 651 (2.4).

MS (EI): $\mathrm{m} / \mathrm{z}=890.2[\mathrm{M}]^{+\bullet}(100 \%)$ for $\mathrm{C}_{56} \mathrm{H}_{42} \mathrm{~N}_{8} \mathrm{O}_{4}$.

\section{zinc(II)-meso- $\alpha-5,10,15-\beta-20$-tetrakis-[2-(acryloylamino)phenyl]-porphyrin (1Zn)}

To a solution of $\mathbf{1 H}_{\mathbf{2}}(200 \mathrm{mg}, 0.22 \mathrm{mmol})$ in methanol $(15 \mathrm{~mL})$ was added zinc acetate in excess with sodium acetate. The mixture was stirred overnight at room temperature, then solvent was removed under vacuo. The resulting powder was dissolved in $\mathrm{CHCl}_{3}$ and directly loaded on a silica gel chromatography column. The expected compound eluted with 5\% $\mathrm{MeOH} / \mathrm{CHCl}_{3}$ was obtained in $90 \%$ yield $(187 \mathrm{mg})$.

MS (EI): $\mathrm{m} / \mathrm{z}=953.8[\mathrm{M}+\mathrm{H}]^{+}(100 \%)$ for $\mathrm{C}_{56} \mathrm{H}_{41} \mathrm{~N}_{8} \mathrm{O}_{4} \mathrm{Zn}$.

\section{meso- $\alpha-5,10,15-t r i s-\{2-(3,3$ ',3'-[N,N',N',-tris-(2-amino-ethylamino)propionylamino]- triphenyl\}- $\beta$-20-[2-(acryloylamino)phenyl]-porphyrin $\left(2 \mathrm{H}_{2}\right)$}

A $250 \mathrm{~mL}$ two neck round bottom flask was charged with $\mathbf{1 H}_{\mathbf{2}}(300 \mathrm{mg}, 0.34 \mathrm{mmol})$ dissolved in $150 \mathrm{~mL}$ of a mixture $\mathrm{CHCl}_{3} / \mathrm{MeOH}(1 / 5)$ degassed during $2 \mathrm{~h}$. The solution was heated at $55^{\circ} \mathrm{C}$, then with a syringe tris $(2$-aminoethyl)amine $(56 \mu \mathrm{L}, 0.37 \mathrm{mmol})$ was added dropwise. Stirring was maintained during $24 \mathrm{~h}$ and then, solvent was evaporated. The product was dissolved in dichloromethane, poured onto a silica gel column and eluted with 0 to $5 \%$ $\mathrm{MeOH} / \mathrm{CH}_{2} \mathrm{Cl}_{2} / \mathrm{NH}_{3 \mathrm{~g}}$ to afford compound $\mathbf{2} \mathbf{H}_{2}$ in $12.5 \%$ yield (44 mg).

${ }^{1} \mathrm{H}$ NMR $\left(500 \mathrm{MHz}, \mathrm{CDCl}_{3}, 323 \mathrm{~K}\right): \delta 11.29(\mathrm{~s}, 1 \mathrm{H}), 10.91(\mathrm{~s}, 2 \mathrm{H}), 8.96-8.80(\mathrm{~m}, 13 \mathrm{H}), 7.91$ (t, $2 \mathrm{H}, \mathrm{J}=8.3 \mathrm{~Hz}), 7.83(\mathrm{td}, 3 \mathrm{H}, \mathrm{Jo}=8.0 \mathrm{~Hz}, \mathrm{Jm}=1.5 \mathrm{~Hz}), 7.66(\mathrm{~d}, 2 \mathrm{H}, \mathrm{J}=7.0 \mathrm{~Hz}), 7.58(\mathrm{td}$, $2 \mathrm{H}, \mathrm{Jo}=7.5 \mathrm{~Hz}, \mathrm{Jm}=1.5 \mathrm{~Hz}), 7.39(\mathrm{~m}, 2 \mathrm{H}), 6.74(\mathrm{~s}, 1 \mathrm{H}), 5.75(\mathrm{~d}, 1 \mathrm{H}, \mathrm{J}=16.5 \mathrm{~Hz}), 5.00(\mathrm{~m}$, $2 \mathrm{H}), 2.12-1.92(\mathrm{~m}, 12 \mathrm{H}), 1.30(\mathrm{~m}, 3 \mathrm{H}), 0.67(\mathrm{~m}, 2 \mathrm{H}), 0.23(\mathrm{~m}, 2 \mathrm{H}),-0.20(\mathrm{~m}, 2 \mathrm{H}),-1.12(\mathrm{~m}$, $2 \mathrm{H}),-1.33(\mathrm{~m}, 2 \mathrm{H}),-1.62(\mathrm{~m}, 2 \mathrm{H}),-2.61(\mathrm{~s}, 2 \mathrm{H})$.

${ }^{13} \mathrm{C}$ NMR $\left(125 \mathrm{MHz}\right.$, pyridine- $\left.d_{5}, 323 \mathrm{~K}\right): \delta 172.0,170.1,165.4,139.8,139.1,138.9,136.9$, $136.7,134.7,132.9,131.3,130.9,130.6,130.2,129.2,127.8,123.5,123.0,122.8,122.2$, $121.8,121.6,117.9,117.5,114.2,46.7,43.7,40.7,39.1,35.2,30.8,30.1,29.3$.

UV-vis: $\lambda_{\text {abs }}\left(\mathrm{CHCl}_{3}\right)\left(\varepsilon, \mathrm{mM}^{-1} \mathrm{~cm}^{-1}\right) 423$ (202.6), 517 (11.7), 522 (3.3), 591 (4.1), 651 (2.4).

MS (EI): $\mathrm{m} / \mathrm{z}=1037.5[\mathrm{M}+\mathrm{H}]^{+}(100 \%)$ for $\mathrm{C}_{62} \mathrm{H}_{61} \mathrm{~N}_{12} \mathrm{O}_{4}$. 


\section{benzyl-pyridin-3-ylmethyl-amine}

Under argon atmosphere, a solution of 3-aminomethylpyridine $(1.5 \mathrm{~mL}, 15 \mathrm{mmol})$ in methanol $(20 \mathrm{~mL})$ degassed with argon during $1 \mathrm{~h}$ was added dropwise to a solution of benzaldehyde $(1.8 \mathrm{~mL}, 18 \mathrm{mmol})$ with a syringe. After stirring for $2 \mathrm{~h}$, the vessel was plunged in an ice bath until the temperature of the solution reached $0^{\circ} \mathrm{C}$. the Schiff's base was reduced with several portions of $\mathrm{NaBH}_{4}(0.7 \mathrm{~g}, 18 \mathrm{mmol})$ at $0^{\circ} \mathrm{C}$. Then, the mixture was stirred at room temperature overnight. The solvent was concentrated under vacuo and $10 \mathrm{~mL}$ of water were added and extracted with ether $(2 \times 20 \mathrm{~mL})$. The organic layer was washed with brine $(2 \times 10 \mathrm{~mL})$. The desired product was extracted with $\mathrm{HCl} 1 \mathrm{~N}(20 \mathrm{~mL})$, washed with ether $(2 \times 10 \mathrm{~mL})$ followed by basification to $\mathrm{pH} 9$ with $\mathrm{K}_{2} \mathrm{CO}_{3}$ and extraction of the desired compound by ether $(2 \times 20 \mathrm{~mL})$. The organic layer was dried over $\mathrm{MgSO}_{4}$ and concentrated under vacuo. Purification by flash column chromatography $\left(1 \% \mathrm{MeOH} / \mathrm{CH}_{2} \mathrm{Cl}_{2}\right)$ afforded a yellow oil $(1.8 \mathrm{~g}, 50 \%)$.

${ }^{1} \mathrm{H}$ NMR $\left(500 \mathrm{MHz}, \mathrm{CDCl}_{3}, 323 \mathrm{~K}\right): \delta 8.50(\mathrm{~d}, 1 \mathrm{H}, \mathrm{J}=2.0 \mathrm{~Hz}), 8.52(\mathrm{dd}, 1 \mathrm{H}, \mathrm{Jo}=5.0 \mathrm{~Hz}, \mathrm{Jm}$ $=1.5 \mathrm{~Hz}), 7.70(\mathrm{dd}, 1 \mathrm{H}, \mathrm{Jo}=7.5 \mathrm{~Hz}, \mathrm{Jm}=1.5 \mathrm{~Hz}), 7.34(\mathrm{~m}, 4 \mathrm{H}), 7.27(\mathrm{~m}, 1 \mathrm{H}), 7.25(\mathrm{~d}, 1 \mathrm{H}, \mathrm{J}$ $=5.0 \mathrm{~Hz}), 3.84(\mathrm{~s}, 4 \mathrm{H}), 1.76(\mathrm{~s}, 1 \mathrm{H})$.

MS (EI): $\mathrm{m} / \mathrm{z}=198.0[\mathrm{M}]^{+\bullet}(100 \%)$ for $\mathrm{C}_{13} \mathrm{H}_{14} \mathrm{~N}_{2}$.

\section{meso- $\alpha-5,10,15-t r i s-\{2-(3,3$ ',3'-[N,N',N''-tris-(2-amino-ethylamino)propionylamino]- triphenyl\}- $\beta$-20-(2-\{3-[(pyridin-3-ylmethyl)-amino]-propionylamino\}-phenyl)-porphyrin $\left(3 \mathbf{H}_{2}\right)$}

\section{Route A :}

In a $100 \mathrm{~mL}$ round bottom flask under argon atmosphere, compound $\mathbf{1 H}_{\mathbf{2}}(1 \mathrm{~g}, 1.12 \mathrm{mmol})$ was dissolved in $600 \mathrm{~mL}$ of a mixture $\mathrm{CHCl}_{3} / \mathrm{MeOH}(1 / 5)$ degassed during 2 hours. The solution was heated at $55^{\circ} \mathrm{C}$ and then tris(2-aminoethyl)amine $(170 \mu \mathrm{L}, 1.13 \mathrm{mmol})$ were added in one portion with a syringe. The reaction mixture was stirred during $24 \mathrm{~h}$ and 5 equivalents of 3 -aminomethylpyridine $(305 \mu \mathrm{L}, 5.65 \mathrm{mmol})$ were added in on portion. Stirring was maintained during $24 \mathrm{~h}$ and then, the mixture was dried under vacuo. The resulting powder was dissolved in dichloromethane and loaded on a silica gel chromatography column. The expected compound eluted with $4 \% \mathrm{MeOH} / \mathrm{CHCl}_{3} / \mathrm{NH}_{3 \mathrm{~g}}$ was obtained in $12 \%$ yield (160 mg).

\section{Route B :}

In a $100-\mathrm{mL}$ bottom flask under argon atmosphere, compound $\mathbf{2} \mathbf{H}_{2}(0.17 \mathrm{~g}, 0.16 \mathrm{mmol})$ was dissolved in $48 \mathrm{~mL}$ of a mixture $\mathrm{CHCl}_{3} / \mathrm{MeOH}(1 / 5)$ degassed during 2 hours. The solution was heated at $55^{\circ} \mathrm{C}$ and then 5 equivalents of 3 -aminomethylpyridine $(75 \mu \mathrm{L}, 0.80 \mathrm{mmol})$ was added. Stirring was maintained during $24 \mathrm{~h}$ and the mixture was dried under vacuo. The resulting powder was dissolved in chloroform and loaded on a silica gel chromatography column. The expected compound eluted with $4 \% \mathrm{MeOH} / \mathrm{CHCl}_{3} / \mathrm{NH}_{3 \mathrm{~g}}$ was obtained in $12 \%$ yield (20 mg).

${ }^{1} \mathrm{H}$ NMR $\left(500 \mathrm{MHz}, \mathrm{CDCl}_{3}, 323 \mathrm{~K}\right) \delta 11.27(\mathrm{~s}, 1 \mathrm{H}), 10.96(\mathrm{~s}, 2 \mathrm{H}), 9.31(\mathrm{~s}, 1 \mathrm{H}), 8.95$ (d, 2H, J $=8.5 \mathrm{~Hz}), 8.92(\mathrm{~d}, 2 \mathrm{H}, \mathrm{J}=4.5 \mathrm{~Hz}), 8.86(\mathrm{~m}, 8 \mathrm{H}), 8.68(\mathrm{~d}, 1 \mathrm{H}, \mathrm{J}=8.5 \mathrm{~Hz}), 7.95(\mathrm{dd}, 1 \mathrm{H}, \mathrm{Jo}=$ $7.5 \mathrm{~Hz}, \mathrm{Jm}=1.5 \mathrm{~Hz}$ ), $7.89(\mathrm{td}, 2 \mathrm{H}, \mathrm{Jo}=1.5 \mathrm{~Hz}, \mathrm{Jm}=7.5 \mathrm{~Hz}), 7.83(\mathrm{t}, 3 \mathrm{H}, \mathrm{J}=7.5 \mathrm{~Hz}), 7.67(\mathrm{~d}$, $1 \mathrm{H}, \mathrm{J}=5.0 \mathrm{~Hz}), 7.59(\mathrm{td}, 3 \mathrm{H}, \mathrm{Jo}=1.5 \mathrm{~Hz}, \mathrm{Jm}=7.5 \mathrm{~Hz}), 7.40(\mathrm{t}, 3 \mathrm{H}, 7.5 \mathrm{~Hz}), 6.35(\mathrm{~s}, 1 \mathrm{H})$, $5.83(\mathrm{dd}, 1 \mathrm{H}, \mathrm{Jo}=4.5 \mathrm{~Hz}, \mathrm{Jm}=7.5 \mathrm{~Hz}), 4.50(\mathrm{~d}, 1 \mathrm{H}, \mathrm{J}=7.5 \mathrm{~Hz}), 2.15-1.90(\mathrm{~m}, 10 \mathrm{H}), 1.79(\mathrm{t}$, $2 \mathrm{H}, \mathrm{J}=5.5 \mathrm{~Hz}), 1.69(\mathrm{t}, 2 \mathrm{H}, \mathrm{J}=5.5 \mathrm{~Hz}), 1.33(\mathrm{~m}, 2 \mathrm{H}), 0.91(\mathrm{t}, 1 \mathrm{H}), 0.66(\mathrm{~m}, 2 \mathrm{H}), 0.55$ (brs, 
2H), 0.37 (s, 2H), 0.21 (brs, 2H), -0.19 (brs, 2H), -0.13 (brs, 2H), -1.31(brs, 2H), -1.63 (brs, $2 \mathrm{H}),-2.67(\mathrm{~s}, 2 \mathrm{H})$.

UV-vis: $\lambda_{\text {abs }}\left(\mathrm{CHCl}_{3}\right)\left(\varepsilon, \mathrm{mM}^{-1} \mathrm{~cm}^{-1}\right) 425$ (453.7), 517 (18.6), 552 (4.9), 592 (5.5), 648 (1.8).

HR-MS (ESI): calcd $\mathrm{m} / \mathrm{z}=1145.5626$ for $\mathrm{C}_{68} \mathrm{H}_{69} \mathrm{~N}_{14} \mathrm{O}_{4}$, found $: \mathrm{m} / \mathrm{z}=1145.5632[\mathrm{M}+\mathrm{H}]^{+}$.

zinc(II)-meso- $\alpha-5,10,15-t r i s-\left\{2-\left(3,3,3, '-\left[N, N^{\prime}, N\right.\right.\right.$,'-tris-(2-amino-

ethylamino)propionylamino]-triphenyl $\}-\beta-20-(2-\{3-[($ pyridin-3-ylmethyl)-amino]propionylamino\}-phenyl)-porphyrin (3Zn)

To a solution of porphyrin $\mathbf{3} \mathbf{H}_{\mathbf{2}}(20 \mathrm{mg}, 0.02 \mathrm{mmol})$ in THF $(5 \mathrm{~mL})$ was added zinc chloride in excess. The mixture was stirred overnight at room temperature and then, triethylamine was added (5 drops). The mixture was stirred at room temperature. Solvent was removed under vacuo. The resulting powder dissolved in chloroform and directly loaded on a silica gel chromatography column. The expected compound 3Zn eluted with chloroform saturated in $\mathrm{NH}_{3 \mathrm{~g}}$ was obtained in $95 \%$ yield $(23 \mathrm{mg})$.

${ }^{1} \mathrm{H}$ NMR $\left(500 \mathrm{MHz}, \mathrm{CDCl}_{3}, 323 \mathrm{~K}\right) \delta 11.21(\mathrm{~s}, 1 \mathrm{H}), 10.90(\mathrm{~s}, 2 \mathrm{H}), 8.96(\mathrm{~d}, 2 \mathrm{H}, \mathrm{J}=8.0 \mathrm{~Hz})$, $8.89(\mathrm{~d}, 4 \mathrm{H}, \mathrm{J}=4.5 \mathrm{~Hz}), 8.85(\mathrm{~d}, 2 \mathrm{H}, \mathrm{J}=4.5 \mathrm{~Hz}), 8.79(\mathrm{~d}, 4 \mathrm{H}, \mathrm{J}=4.5 \mathrm{~Hz}), 8.74(\mathrm{~s}, 1 \mathrm{H}), 8.64$ $(\mathrm{d}, 1 \mathrm{H}, \mathrm{J}=8.0 \mathrm{~Hz}), 7.84-7.74(\mathrm{~m}, 4 \mathrm{H}), 7.65(\mathrm{dd}, 1 \mathrm{H}, \mathrm{Jo}=7.5 \mathrm{~Hz}, \mathrm{Jm}=1.5 \mathrm{~Hz}), 7.49(\mathrm{dd}, 1 \mathrm{H}$, $\mathrm{Jo}=7.5 \mathrm{~Hz}, \mathrm{Jm}=1.5 \mathrm{~Hz}), 7.43(\mathrm{t}, 2 \mathrm{H}, \mathrm{J}=5.0 \mathrm{~Hz}), 7.35-7.39(\mathrm{~m}, 4 \mathrm{H}), 7.26(\mathrm{t}, 2 \mathrm{H}, \mathrm{J}=5.0$ $\mathrm{Hz}), 6.22(\mathrm{~d}, 1 \mathrm{H}, \mathrm{J}=8.0 \mathrm{~Hz}), 5.53(\mathrm{~d}, 1 \mathrm{H}, \mathrm{J}=8.5 \mathrm{~Hz}), 2.58(\mathrm{~s}, 2 \mathrm{H}), 2.45(\mathrm{~d}, 1 \mathrm{H}, \mathrm{J}=5.0 \mathrm{~Hz})$, $2.23(\mathrm{~m}, 4 \mathrm{H}), 2.13-1.89(\mathrm{~m}, 8 \mathrm{H}), 1.75(\mathrm{~m}, 2 \mathrm{H}), 1.55$ (brs, 4H), $0.61(\mathrm{~m}, 2 \mathrm{H}), 0.5(\mathrm{t}, 2 \mathrm{H}, \mathrm{J}=$ $5.0 \mathrm{~Hz}), 0.16(\mathrm{t}, 2 \mathrm{H}, \mathrm{J}=5.0 \mathrm{~Hz}),-0.24(\mathrm{~m}, 2 \mathrm{H}),-1.19(\mathrm{~m}, 2 \mathrm{H}),-1.41(\mathrm{~m}, 2 \mathrm{H})$.

UV-vis: $\lambda_{\text {abs }}\left(\mathrm{CHCl}_{3}\right)\left(\varepsilon, \mathrm{mM}^{-1} \mathrm{~cm}^{-1}\right) 414$ (26.2) 433 (413.8), 527 (2.3), 527 (2.3), 563 (12.5), 605 (3.2).

HR-MS (ESI): calcd m/z = 1207.4761 for $\mathrm{C}_{68} \mathrm{H}_{67} \mathrm{~N}_{14} \mathrm{O}_{4} \mathrm{Zn}$, found: $\mathrm{m} / \mathrm{z}=1207.4731[\mathrm{M}+\mathrm{H}]^{+}$.

\section{iron(II)-meso- $\alpha-5,10,15-t r i s-\left\{2-\left(3,3,3, '-\left[N, N^{\prime}, N^{\prime \prime}\right.\right.\right.$-tris-(2-amino-} ethylamino)propionylamino]-triphenyl $\}-\beta-20-(2-\{3-[($ pyridin-3-ylmethyl)-amino]propionylamino\}-phenyl)-porphyrin (3Fe)

In a dry box, to a solution of porphyrin $\mathbf{3} \mathbf{H}_{\mathbf{2}}(10 \mathrm{mg}, 0.009 \mathrm{mmol})$ in THF $(6 \mathrm{~mL})$ was added iron(II) bromide. The mixture was stirred overnight at $65^{\circ} \mathrm{C}$, then solvent was removed under vacuo. The resulting powder was dissolved in benzene/ $\mathrm{MeOH}(5 / 1)$ and directly loaded on a silica gel chromatography column. The expected compound 3 Fe was eluted with a gradient of methanol in benzene with triethylamine.

HR-MS (ESI): calcd 1198.4741 for $\mathrm{C}_{68} \mathrm{H}_{66} \mathrm{~N}_{14} \mathrm{O}_{4}{ }^{56} \mathrm{Fe}$ found: $\mathrm{m} / \mathrm{z}=1198,4745[\mathrm{M}]^{+\bullet}$.

UV-vis: Soret ( $\lambda$, toluene): 3Fe: $439 \mathrm{~nm}$; $+\mathrm{O}_{2}: 428 \mathrm{~nm}$.

\section{zinc(II)-meso- $\alpha-5,10,15-t r i s-\left\{2-\left(3,3,3, '-\left[N, N^{\prime}, N^{\prime} '-t r i s-(2-a m i n o-\right.\right.\right.$}

ethylamino)propionylamino]-triphenyl $\}-\beta-20-[2-(3-\{2-[$ bis-(2-amino-ethyl)-amino]ethylamino\}-propionylamino)-phenyl]-porphyrin (4Zn)

In a $100 \mathrm{~mL}$ round bottom flask under argon, $150 \mathrm{mg}(0.16 \mathrm{mmol})$ of $\mathbf{2 Z n}$ were dissolved in $75 \mathrm{~mL}$ of a mixture $\mathrm{CHCl}_{3} / \mathrm{MeOH}(1 / 5)$ degassed during 2 hours. The solution was heated at $55^{\circ} \mathrm{C}$, then tris $(2$-aminoethyl)amine $(51 \mu \mathrm{L}, 0.34 \mathrm{mmol})$ was added in one portion with a syringe. The reaction mixture was stirred during 48 hours and dried under vacuo. The 
resulting powder was dissolved in dichloromethane and loaded on a silica gel chromatography column. The expected compound $4 \mathrm{Zn}$ eluted with $20 \% \mathrm{MeOH} / \mathrm{CHCl}_{3}$ was obtained in $10 \%$ yield $(20 \mathrm{mg})$.

${ }^{1} \mathrm{H}$ NMR (500MHz, DMSO-d 6 , 353K): $\delta 10.81(\mathrm{~s}, 1 \mathrm{H}), 10.68(\mathrm{~s}, 2 \mathrm{H}), 9.93(\mathrm{~s}, 1 \mathrm{H}), 8,75(\mathrm{~d}$, $2 \mathrm{H}, \mathrm{J}=4.5 \mathrm{~Hz}), 8.73(\mathrm{dd}, 1 \mathrm{H}, \mathrm{Jo}=8.5 \mathrm{~Hz}, \mathrm{Jm}=1.0 \mathrm{~Hz}), 8.68(\mathrm{~d}, 2 \mathrm{H}, \mathrm{J}=4.5 \mathrm{~Hz}), 8,66(\mathrm{dd}$, $1 \mathrm{H}, \mathrm{Jo}=8.0 \mathrm{~Hz}, \mathrm{Jm}=1.5 \mathrm{~Hz}), 8.65(\mathrm{~d}, 2 \mathrm{H}, \mathrm{J}=4.5 \mathrm{~Hz}), 8.64(\mathrm{~d}, 2 \mathrm{H}, \mathrm{J}=4.5 \mathrm{~Hz}), 7.77-7.69$ $\left(\mathrm{m}, 8 \mathrm{H}, \mathrm{H}_{\text {aro }}\right), 7.61(\mathrm{dd}, 1 \mathrm{H}, \mathrm{Jo}=7.5 \mathrm{~Hz}, \mathrm{Jm}=1.5 \mathrm{~Hz}$ ), $7.53(\mathrm{dd}, 1 \mathrm{H}, \mathrm{Jo}=7.5 \mathrm{~Hz}, \mathrm{Jm}=1.5$ $\mathrm{Hz}), 7.43-7.35(\mathrm{~m}, 4 \mathrm{H}), 1.98-1.76(\mathrm{~m}, 20 \mathrm{H}), 1.06(\mathrm{t}, 2 \mathrm{H}, \mathrm{J}=5.0 \mathrm{~Hz}), 0.96(\mathrm{t}, 2 \mathrm{H}, \mathrm{J}=7.5 \mathrm{~Hz})$, $0.52(\mathrm{~m}, 4 \mathrm{H}), 0.29(\mathrm{~m}, 2 \mathrm{H}), 0.10(\mathrm{~m}, 2 \mathrm{H}),-0.09(\mathrm{~m}, 4 \mathrm{H}),-0.43(\mathrm{~m}, 4 \mathrm{H}),-0.53(\mathrm{~m}, 4 \mathrm{H}),-1.18$ (m, 2H), -1.64 (m, 2H).

${ }^{13} \mathrm{C}$ NMR (125MHz, DMSO- $\left.d_{6}, 353 \mathrm{~K}\right): \delta 137.4,137.3,136.1,132.2,132.1,131.7,129.3$, 129.1, 123.1, 122.9, 122.4, 122.3, 122.1, 121.9, 51.8, 49.1, 47.5, 44.7, 44.4, 43.9, 43.2, 42.4, 41.9, 36.7, 35.7, 35.2.

MS (EI): $1245.44[\mathrm{M}]^{+\bullet}(100 \%)$ for $\mathrm{C}_{68} \mathrm{H}_{76} \mathrm{~N}_{16} \mathrm{O}_{4} \mathrm{Zn}$.

Equilibrium Measurements. The $\mathrm{O}_{2}$ affinity was measured spectrophotometrically under equilibrium conditions with an Uvikon XL spectrometer. Measurements were performed with toluene solutions thermostated in the spectrometer to $25.0 \pm 0.3{ }^{\circ} \mathrm{C}$ with a Lauda RL6 constant-temperature bath and circulator.

The equilibrium between the metalloporphyrin (MP.B) and $\mathrm{O}_{2}$ can be expressed as follows:

$\mathrm{MP} . \mathrm{B}+\mathrm{O}_{2} \rightleftharpoons \mathrm{K}=$ MP.B.O 2

The equilibrium constant $\mathrm{K}$ is defined by:

$\mathrm{K}=\left[\mathrm{MP} . \mathrm{B} . \mathrm{O}_{2}\right] /[\mathrm{MP} . \mathrm{B}] p\left(\mathrm{O}_{2}\right)$

If $\mathrm{C}_{0}$ is the total concentration of (MP.B), then at $\mathrm{t}=0$ (without dioxygen): $\mathrm{C}_{0}=$ [MP.B]. At a t instant with a dioxygen partial pressure $\left(p\left(\mathrm{O}_{2}\right)\right): \mathrm{C}_{0}=[\mathrm{MP} . \mathrm{B}]+\left[\mathrm{MP} . \mathrm{B} . \mathrm{O}_{2}\right]$.

Then: $\mathrm{K}=\left[\mathrm{MP} . \mathrm{B} . \mathrm{O}_{2}\right] /\left(\left(\mathrm{C}_{0}-\left[\mathrm{MP} . \mathrm{B} . \mathrm{O}_{2}\right]\right) \cdot p\left(\mathrm{O}_{2}\right)\right)$

Only (MP.B) and (MP.B. $\mathrm{O}_{2}$ ) have absorbance in UV-Vis. The Beer-Lambert law can be written as follow: When $\mathrm{t}=0$ : Ao $=\varepsilon_{\text {MP.B.Co }}=\varepsilon_{\text {MP.B.[MP.B }]}+\varepsilon_{\text {MP.B. }}[$ MP.B.O 2$]$

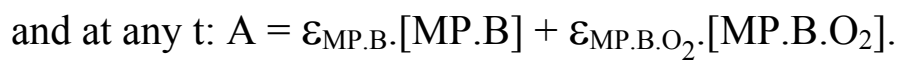

Then $\mathrm{A}-\mathrm{Ao}=\left(\varepsilon_{\text {MP.B.O }}-\varepsilon_{\text {MP.B }}\right)$. [MP.B.O $\left.\mathrm{O}_{2}\right]$ or $\Delta \mathrm{A}=\Delta \varepsilon$. [MP.B. $\left.{ }_{2}\right]$

The equilibrium constant $\mathrm{K}$ could be defined by:

$\mathrm{K}=\Delta \mathrm{A} /\left(\left(\mathrm{C}_{0} \Delta \varepsilon-\Delta \mathrm{A}\right) \cdot p\left(\mathrm{O}_{2}\right)\right)$ or $\mathrm{K}^{-1}=\left(\left(\mathrm{C}_{0} \Delta \varepsilon / \Delta \mathrm{A}\right) \cdot p\left(\mathrm{O}_{2}\right)\right)-p\left(\mathrm{O}_{2}\right)$

The partial pressure of dioxygen $\left(p\left(\mathrm{O}_{2}\right)\right)$ could be defined as: $p\left(\mathrm{O}_{2}\right)=\left(\left(\mathrm{C}_{0} \Delta \varepsilon / \Delta \mathrm{A}\right) \cdot p\left(\mathrm{O}_{2}\right)\right)-\mathrm{K}^{-1}$

This is the equation of a straight line with a slope of $\mathrm{C}_{0} \Delta \varepsilon$ and an intercept of $-1 / \mathrm{K}$. When the metalloporphyrin is half-oxygenated: [MP.B] $=\left[\mathrm{MP} . \mathrm{B} . \mathrm{O}_{2}\right]$ then $1 / \mathrm{K}=\mathrm{P}_{1 / 2}\left(\mathrm{O}_{2}\right)$. The partial pressure of oxygen at which $50 \%$ of metalloporphyrin sites are bound with $\mathrm{O}_{2}$ is defined as $\mathrm{P}_{1 / 2}\left(\mathrm{O}_{2}\right)$.

Determination of $\mathrm{P}_{1 / 2}\left(\mathrm{O}_{2}\right)$ for compound 3Fe

$\mathbf{V O}_{2}$ : volume of pure dioxygen injected in the dilution flask filled with $\mathrm{N}_{2}$ from a dry box maintained under $0.5 \mathrm{ppm}$ of $\mathrm{O}_{2}$.

Vol tonometer: total volume of the tonometer. 
Added volume: represents the effective volume of diluted dioxygen from the dilution flask into the tonometer. 


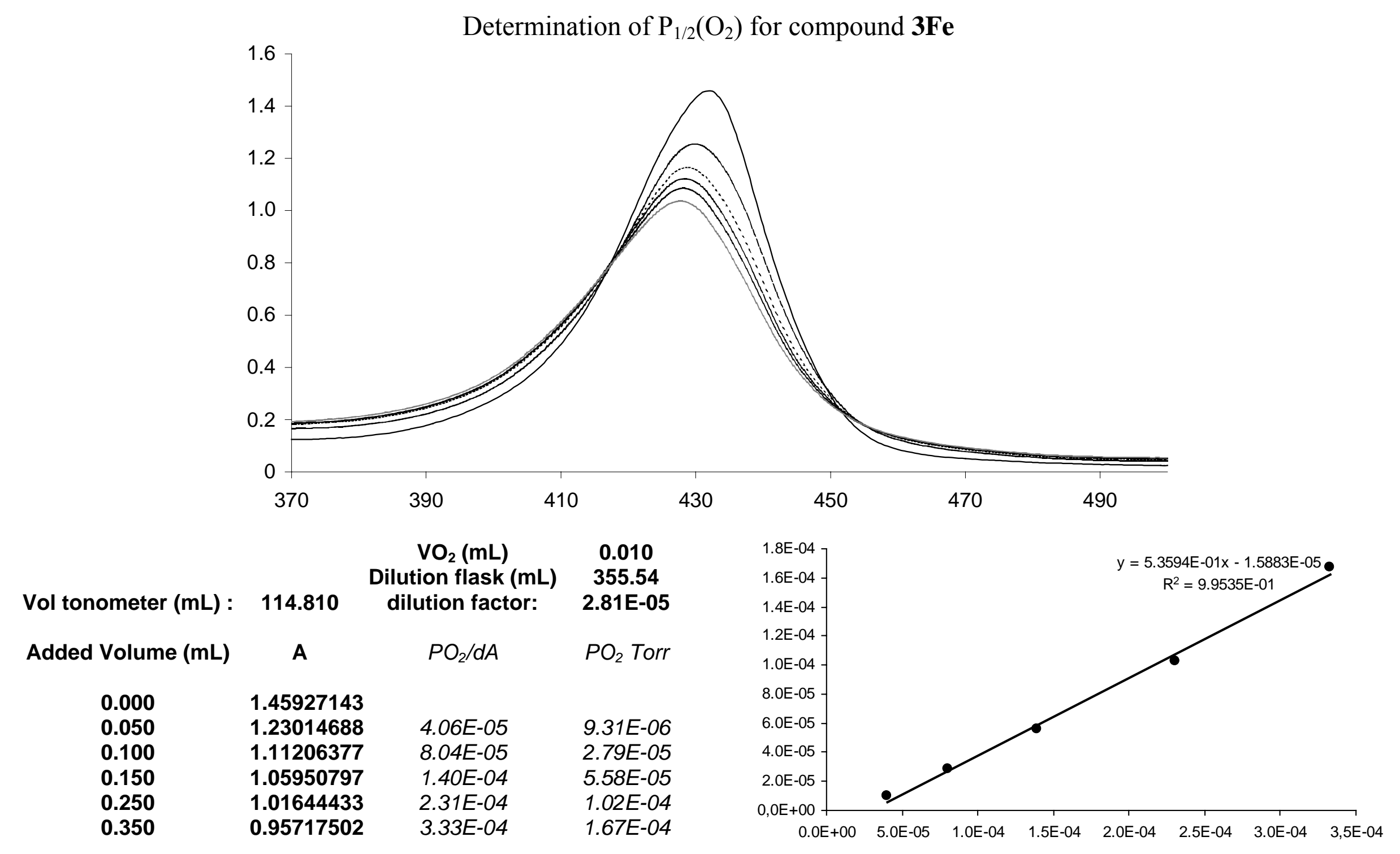




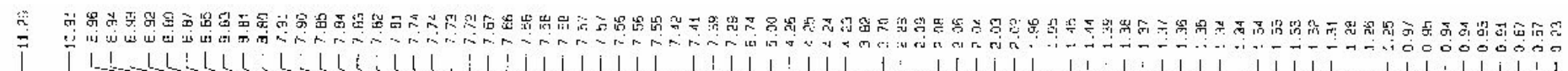
lon

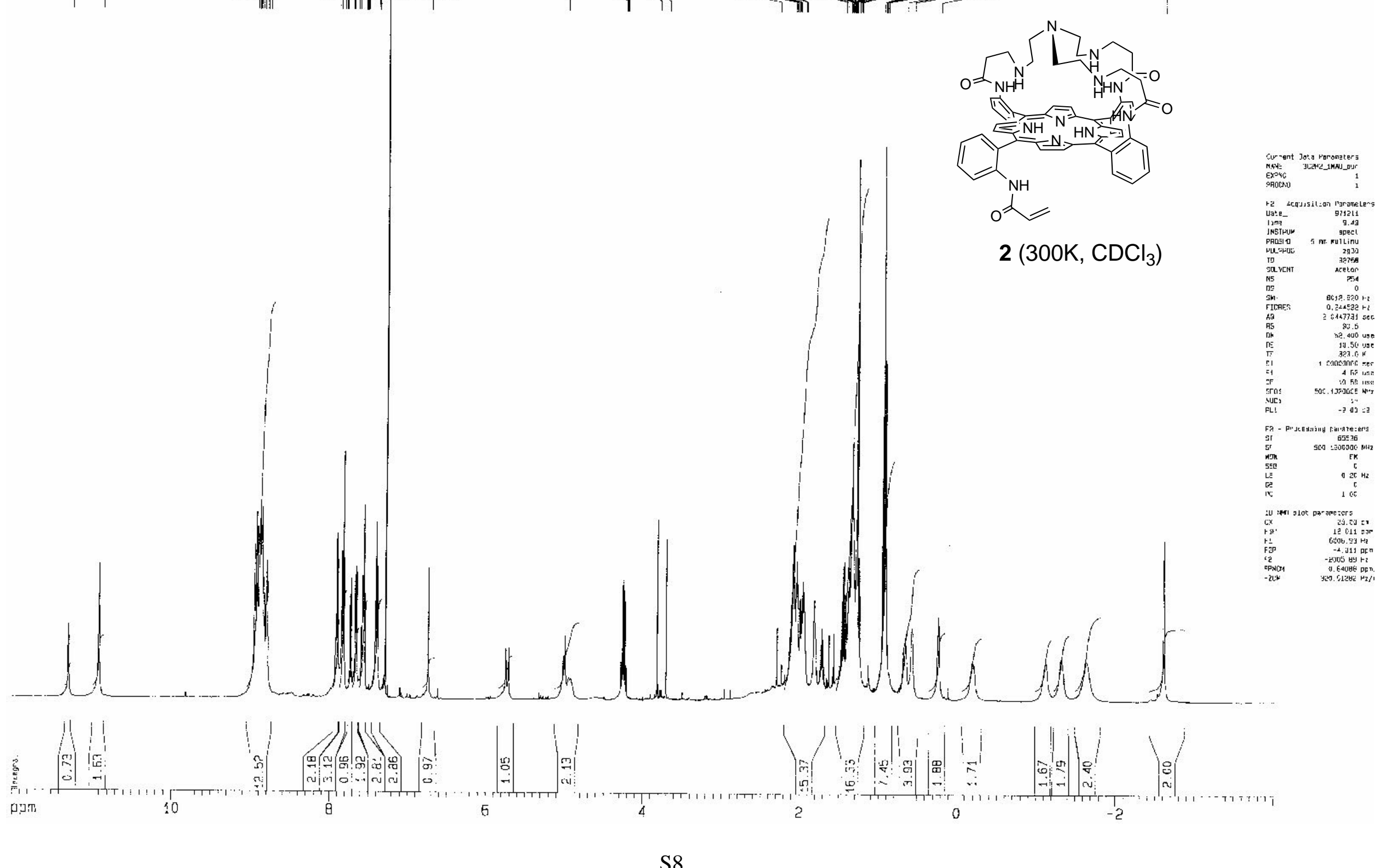




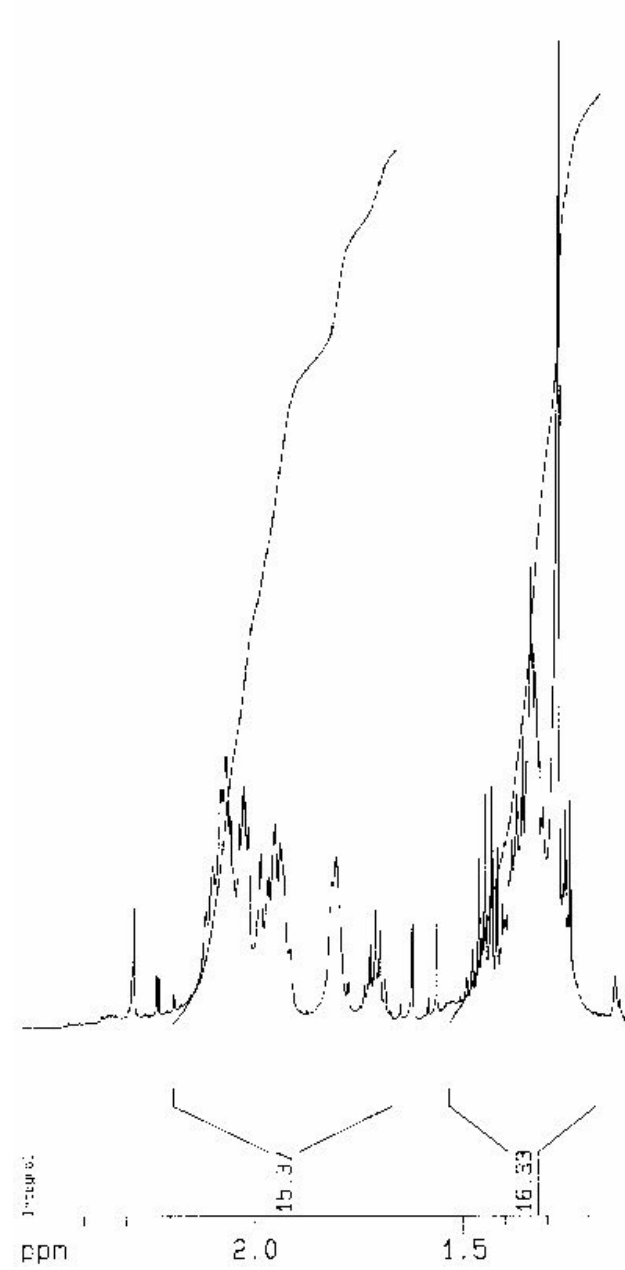

FРГ
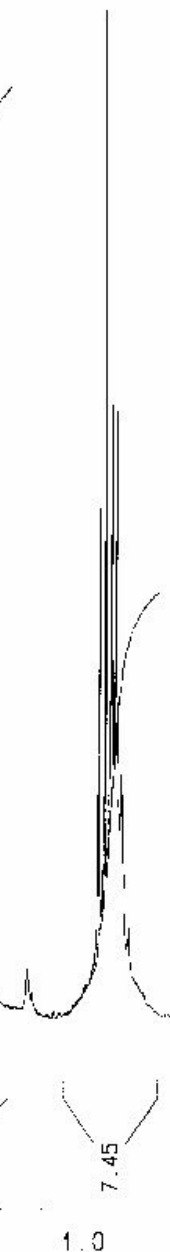

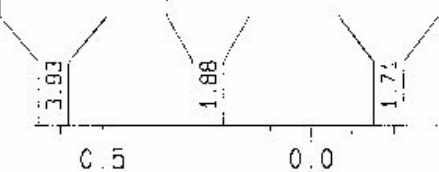

$-0.5$
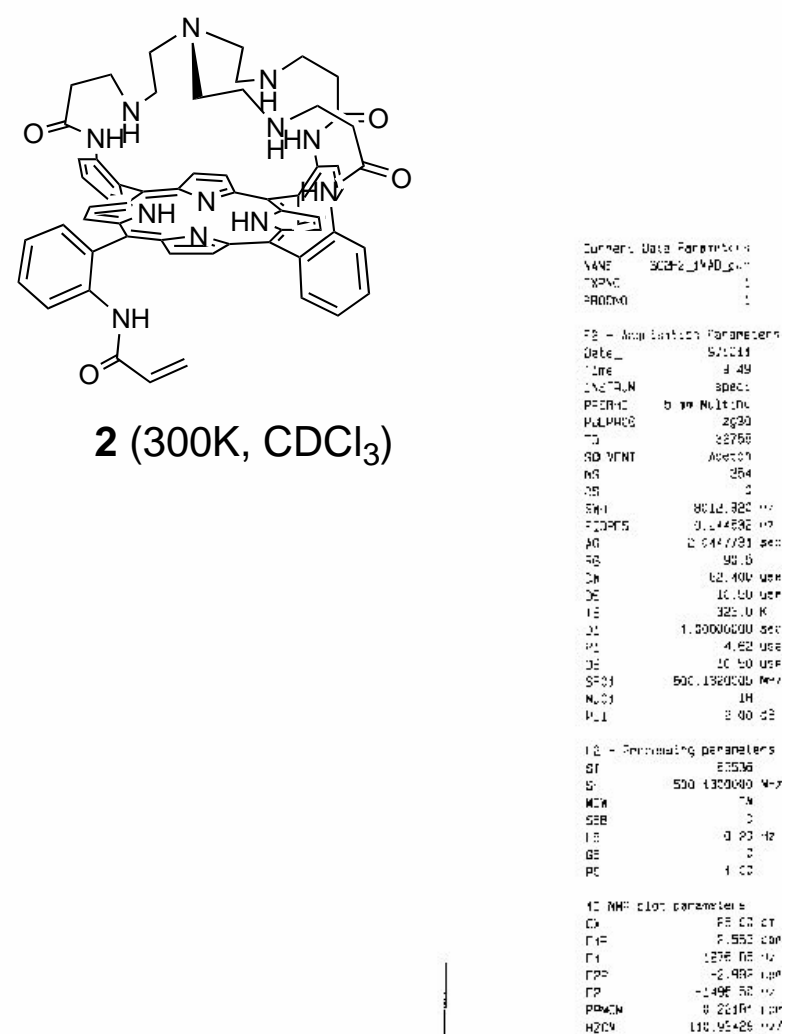


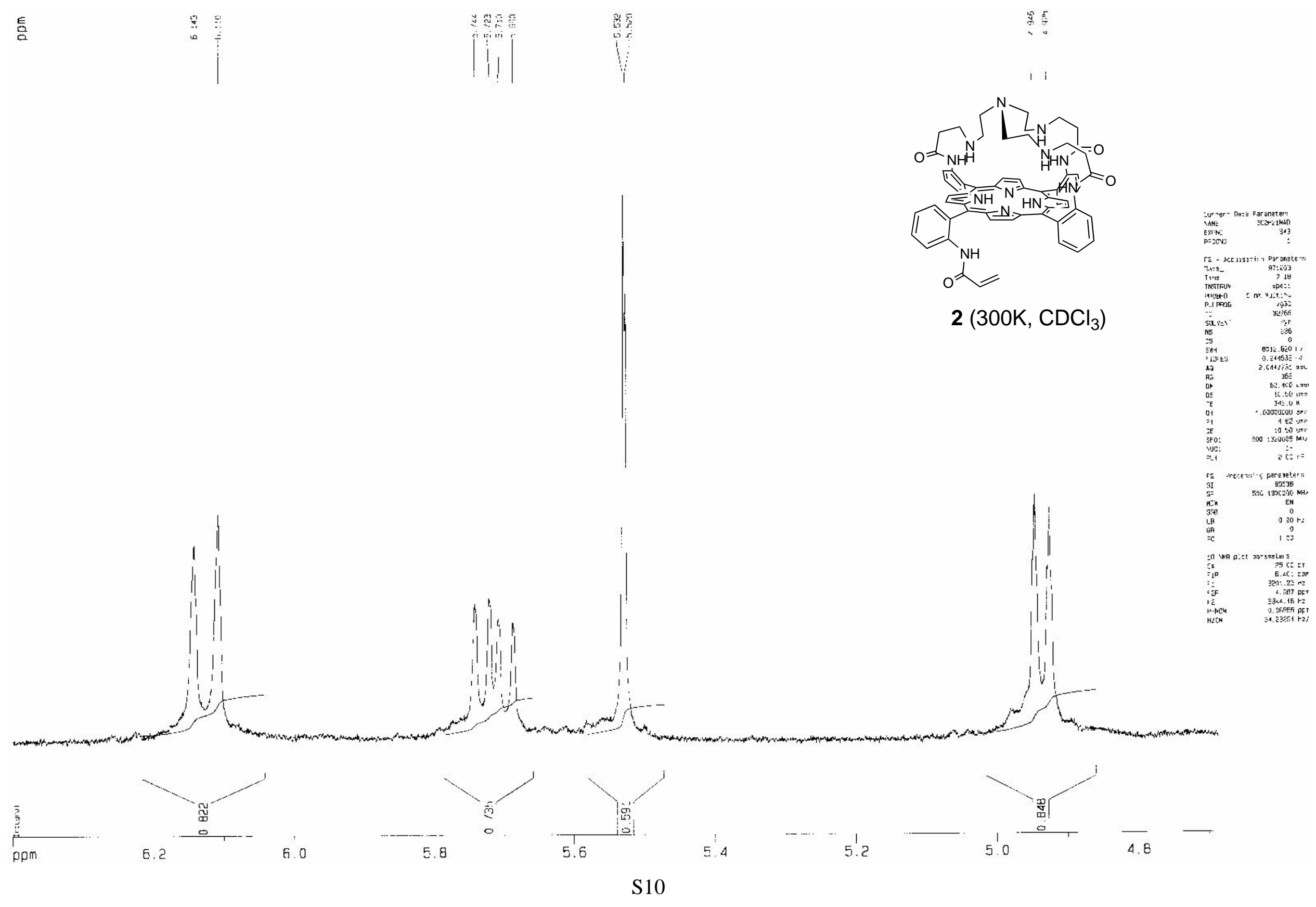



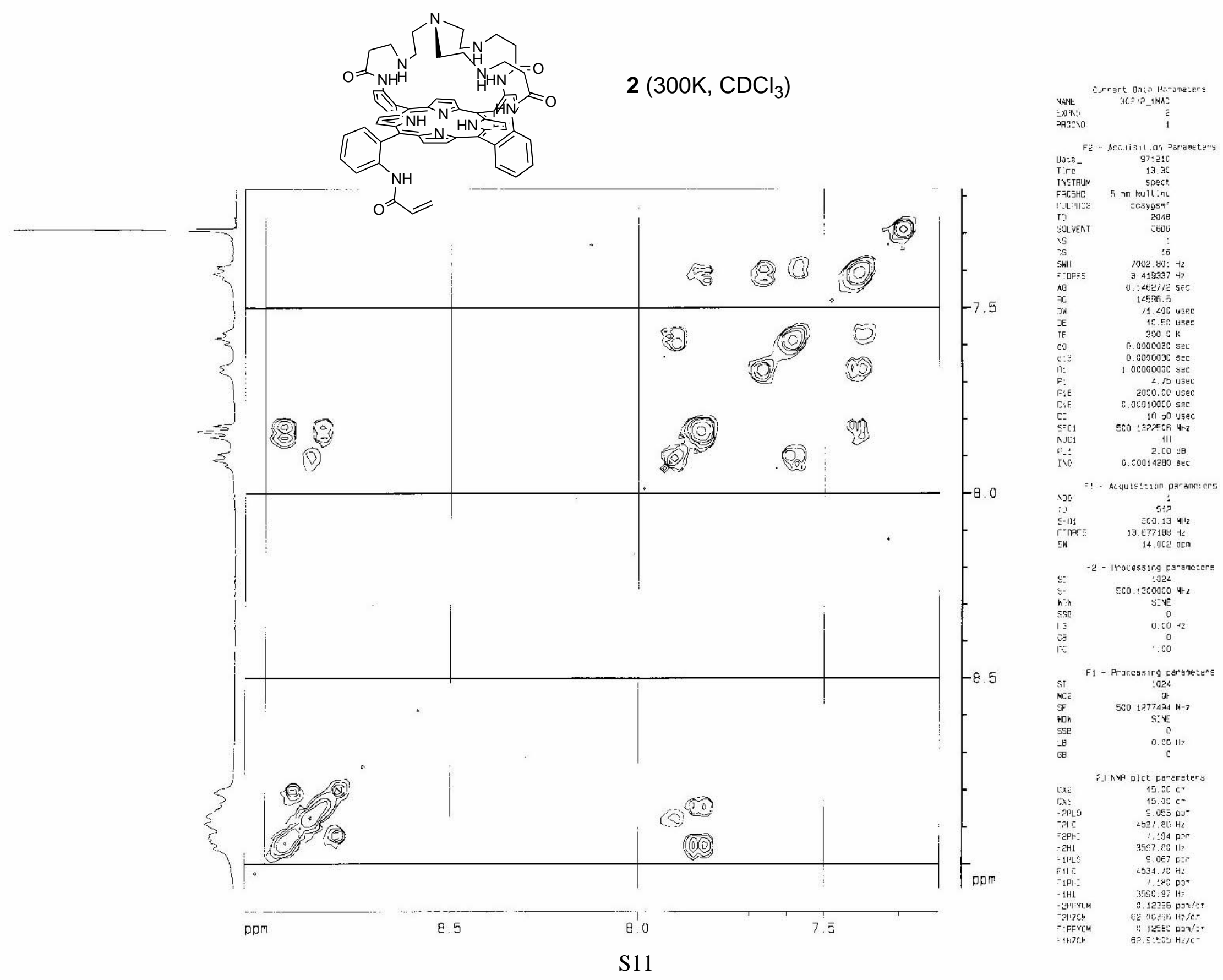
[3. 030

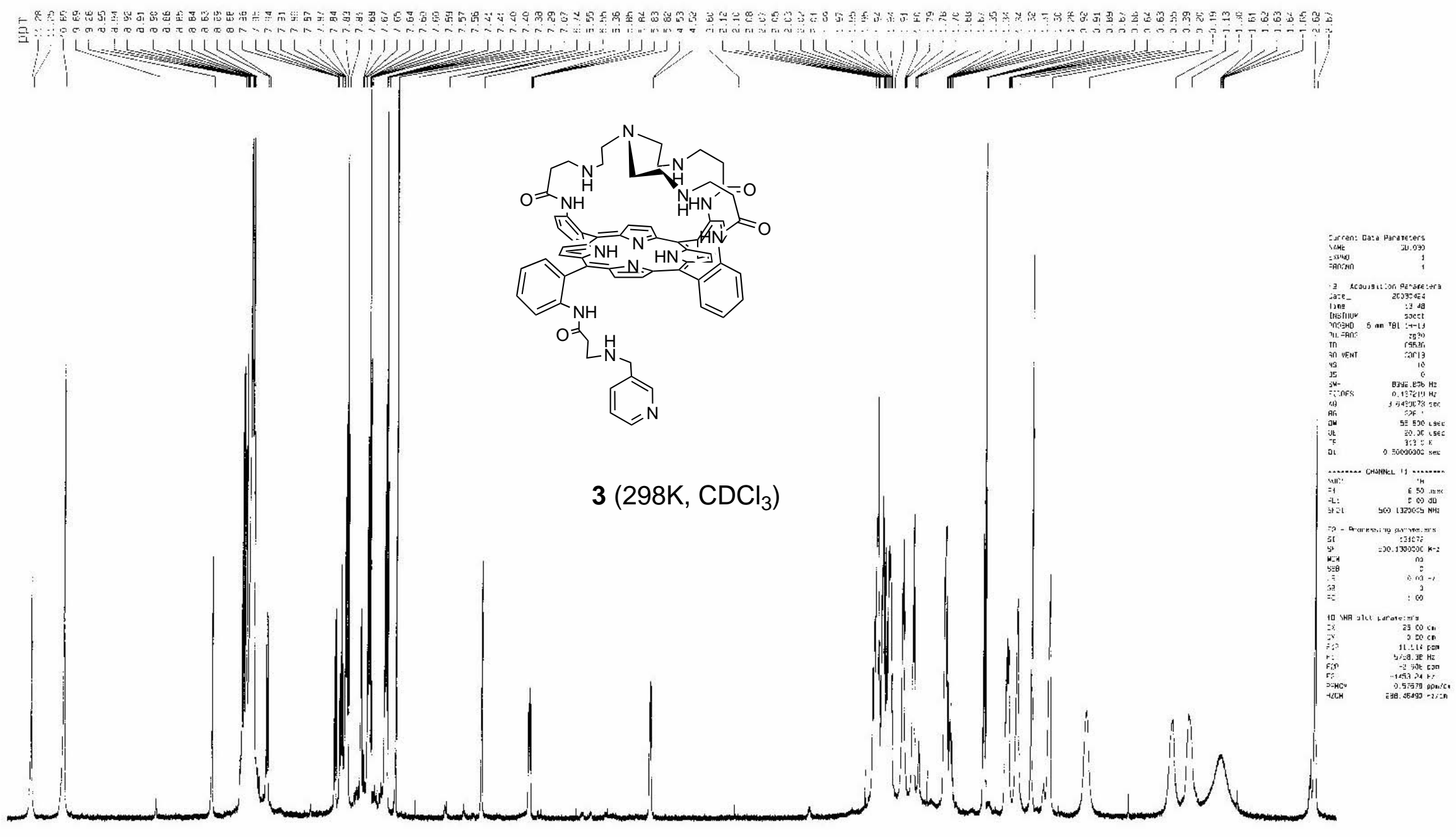


01090

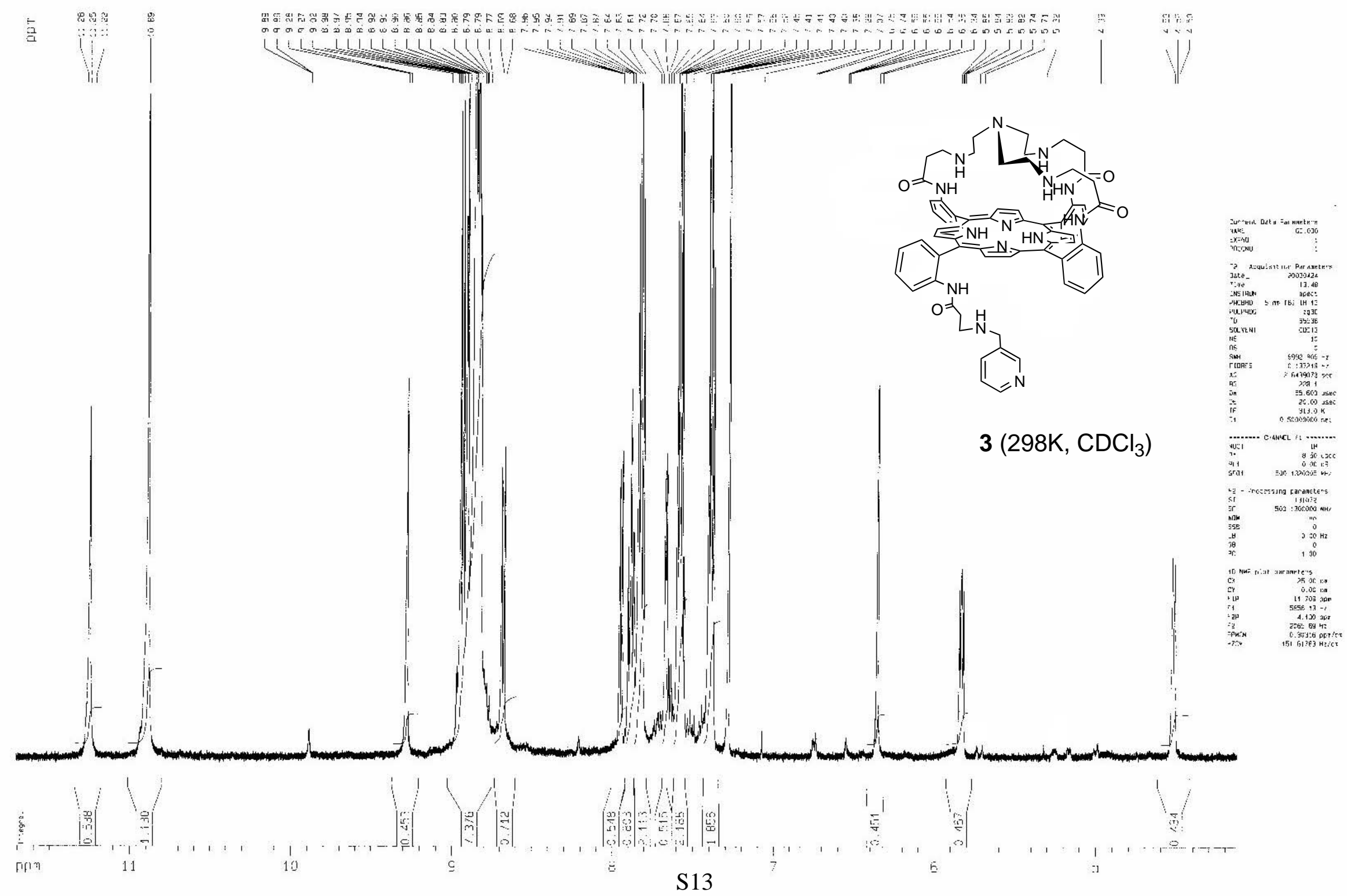


er. 03.
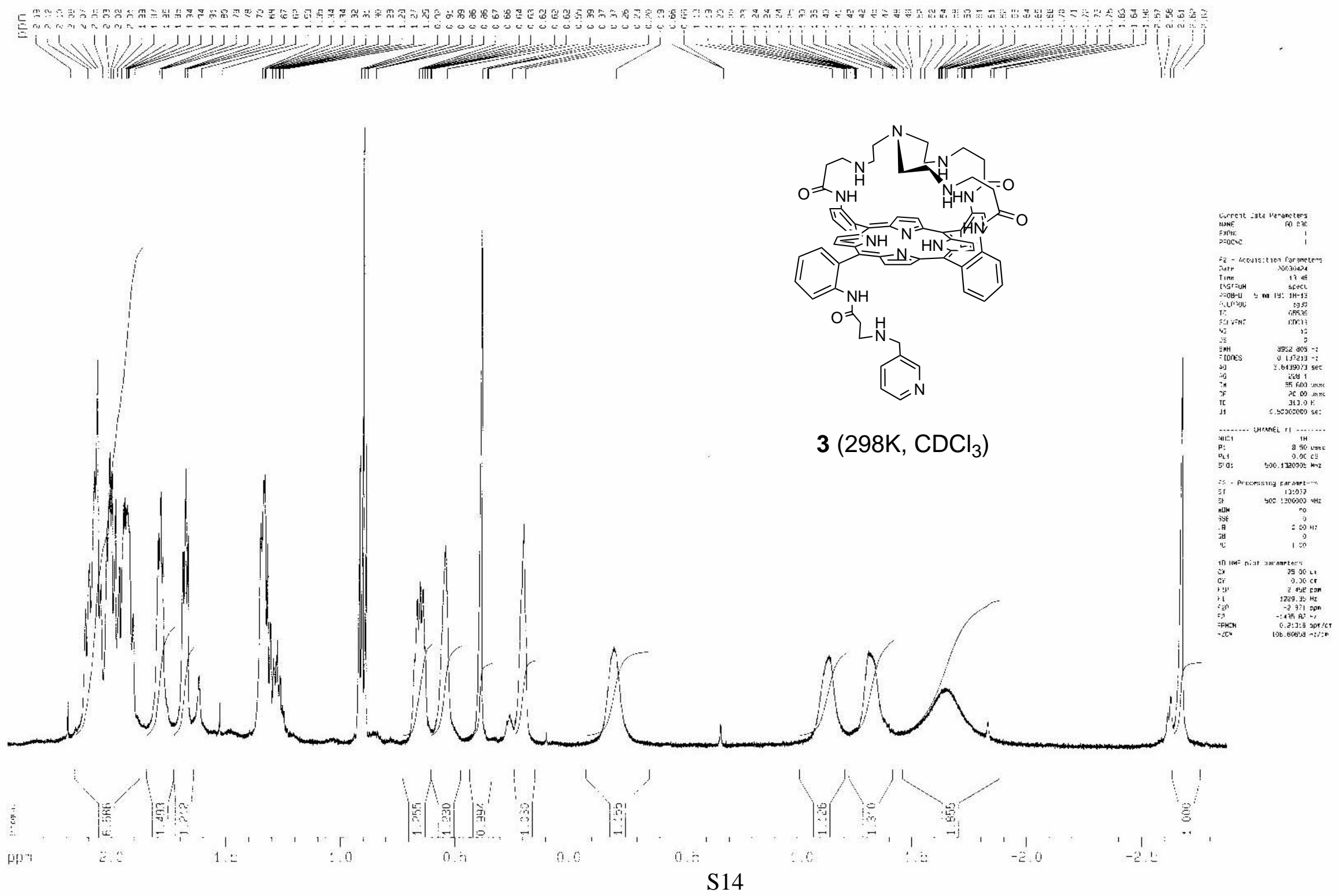


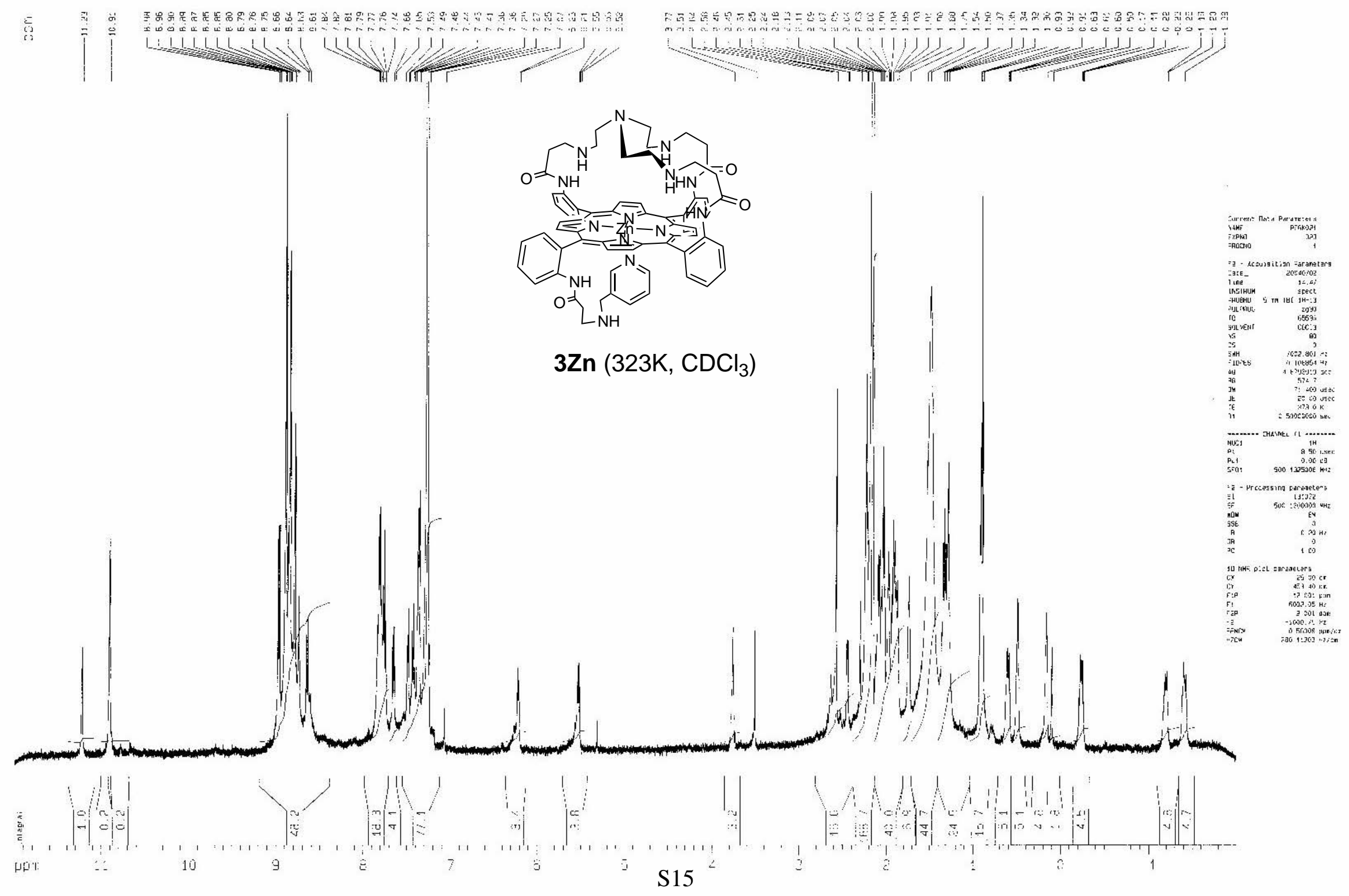




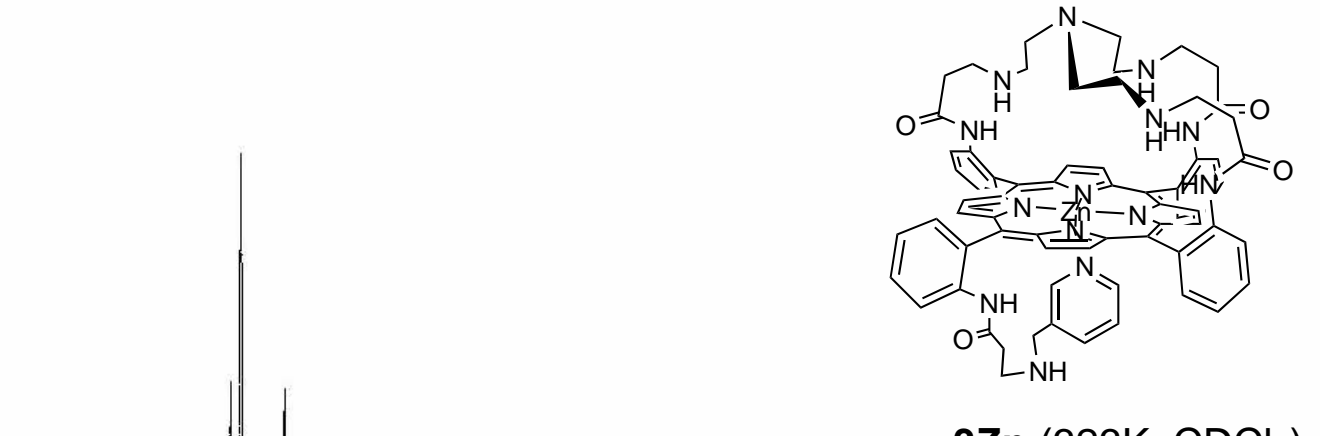

3Zn (323K, $\left.\mathrm{CDCl}_{3}\right)$
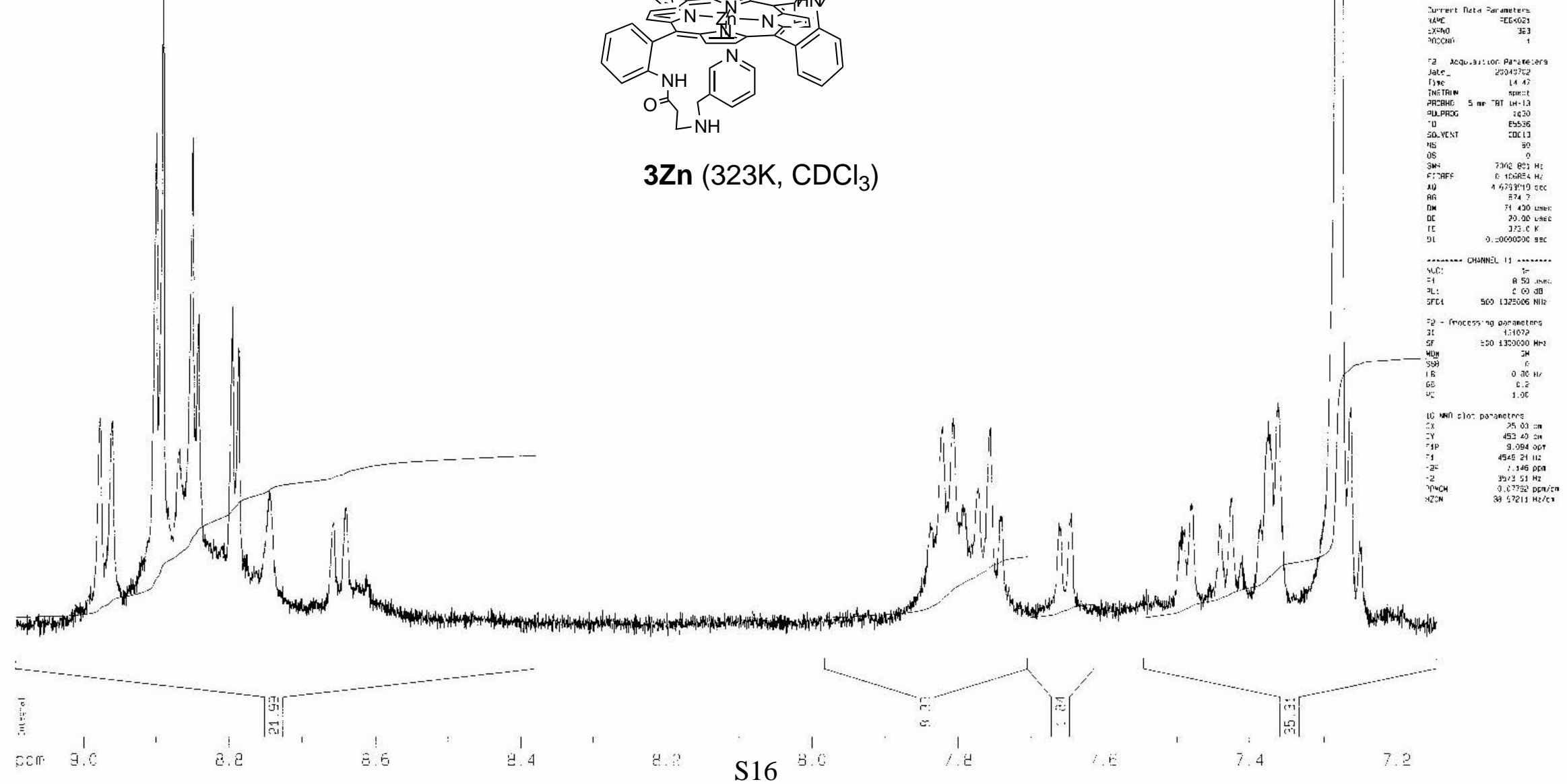


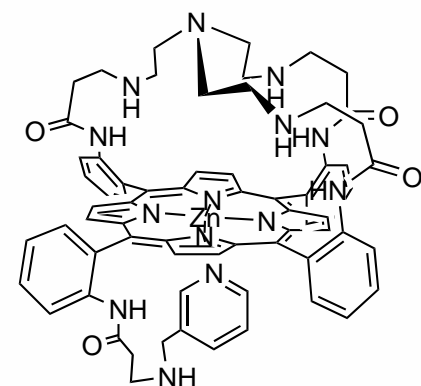

3Zn (323K, $\left.\mathrm{CDCl}_{3}\right)$
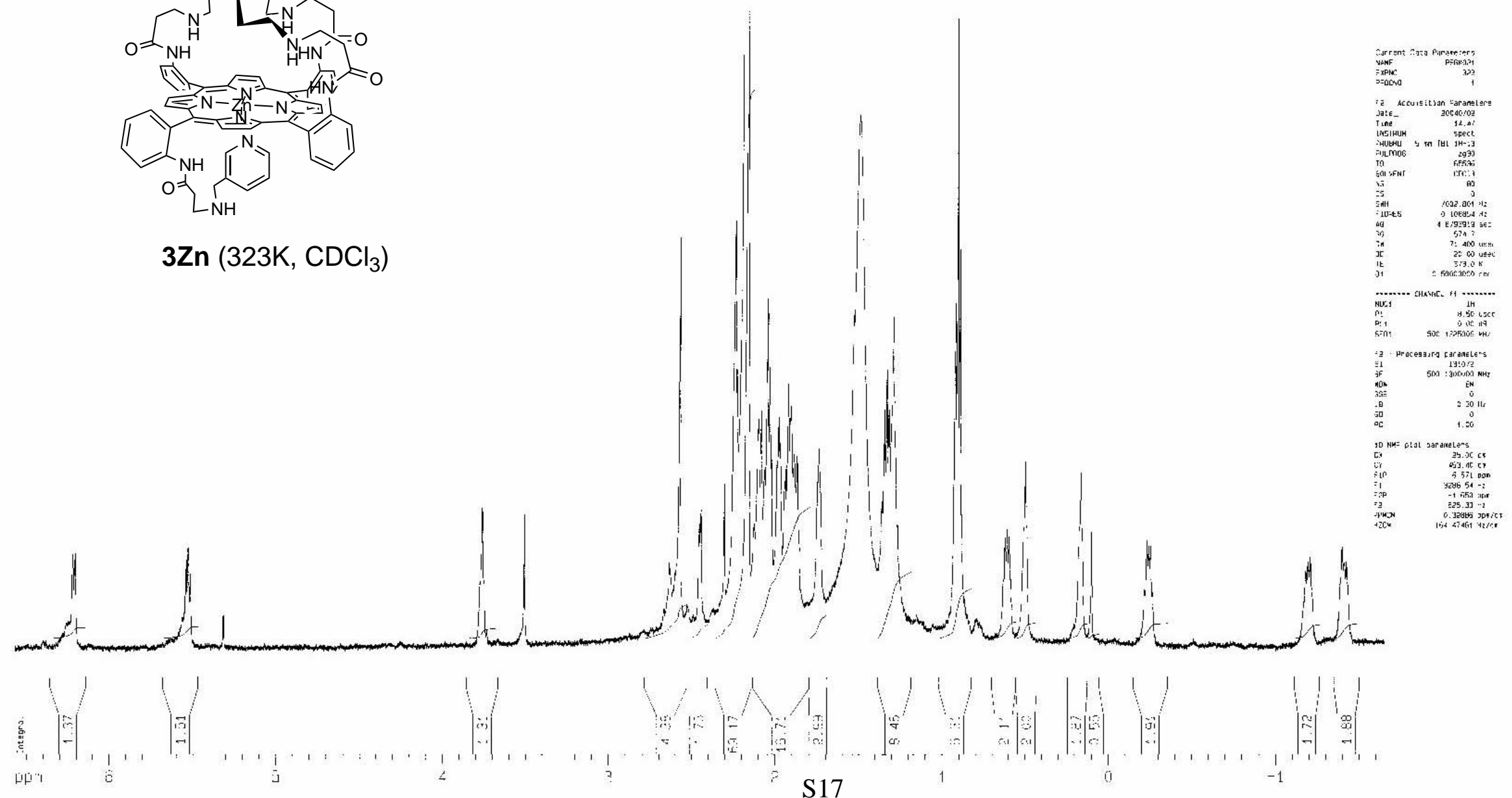
$25 \%$

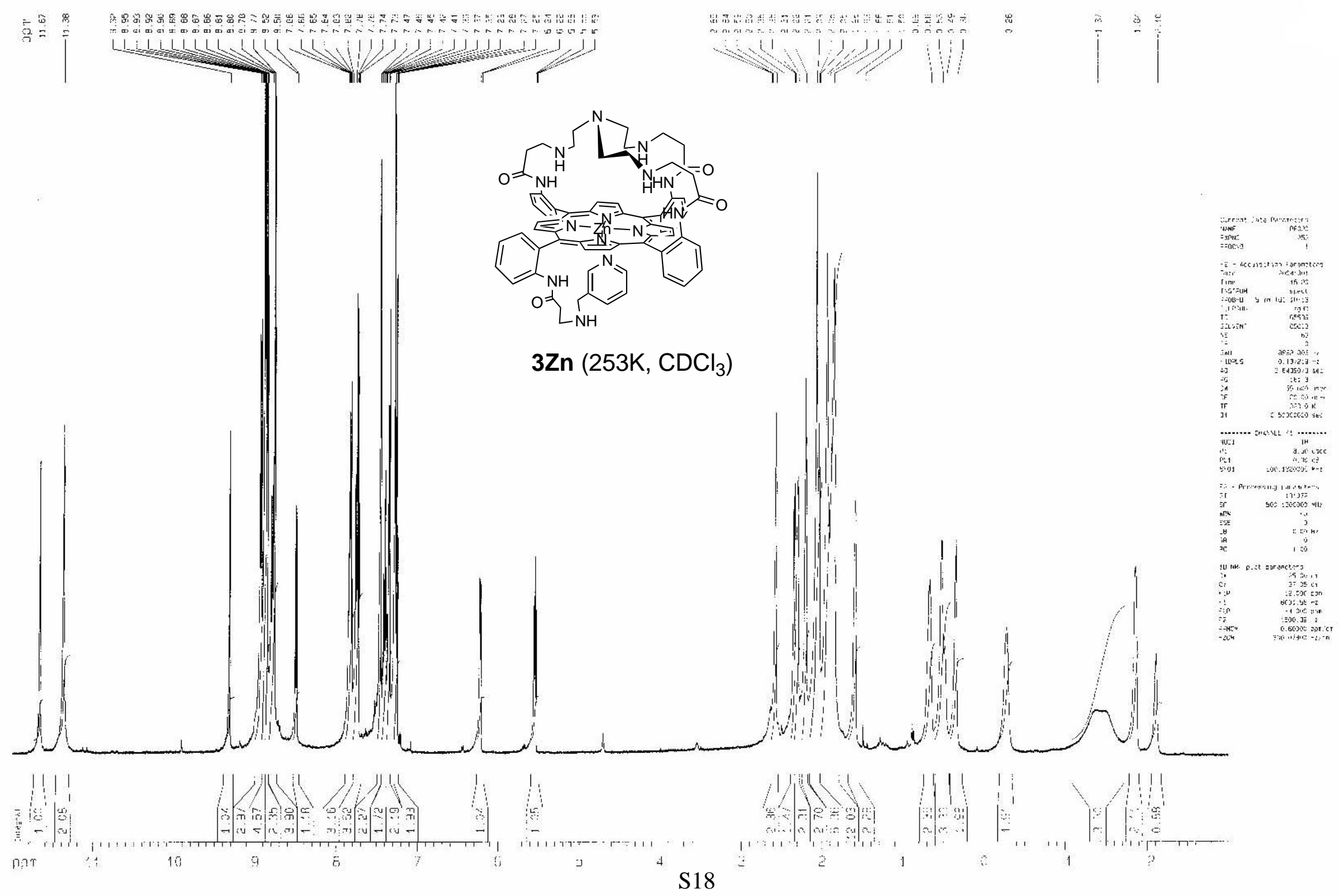




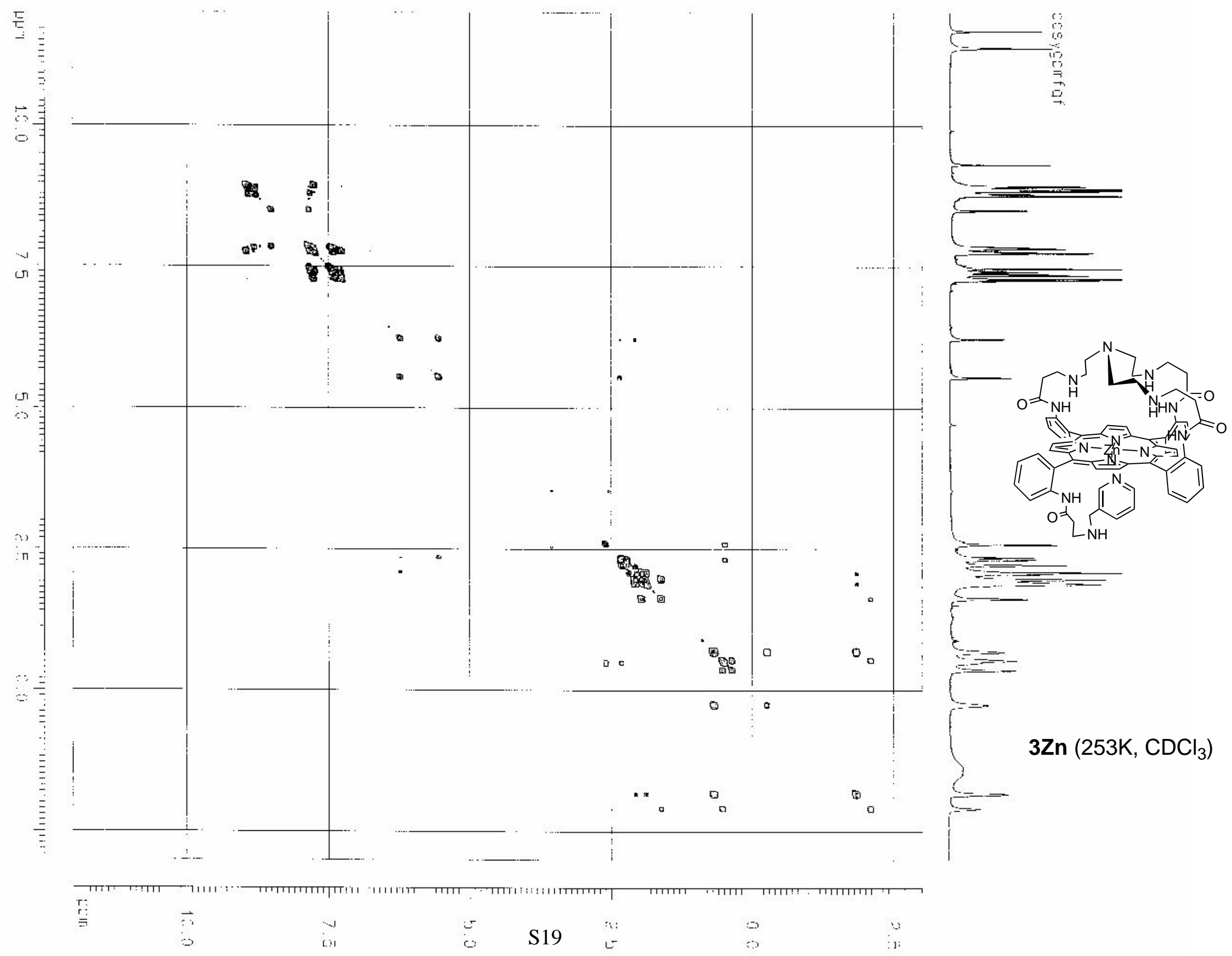




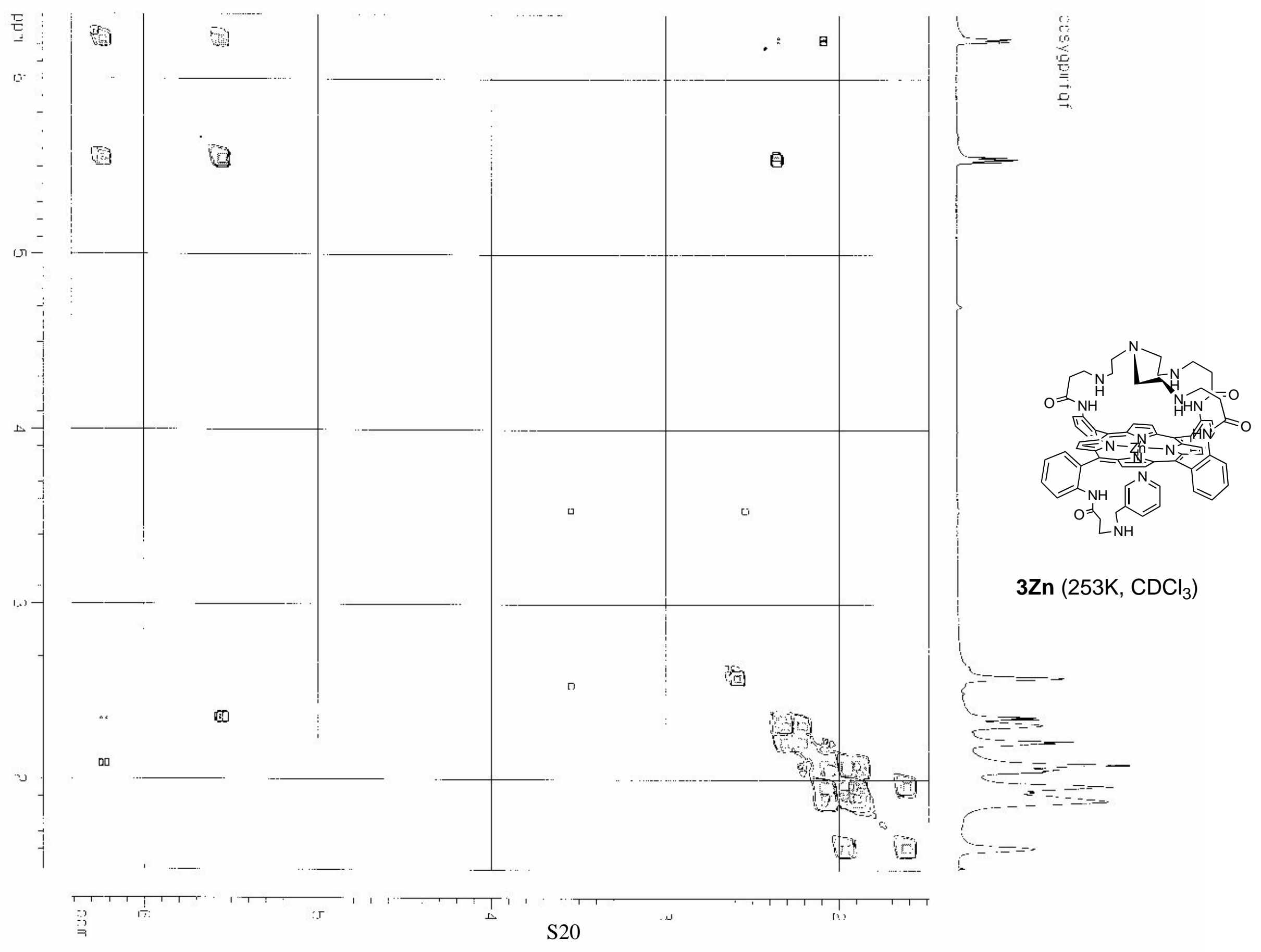




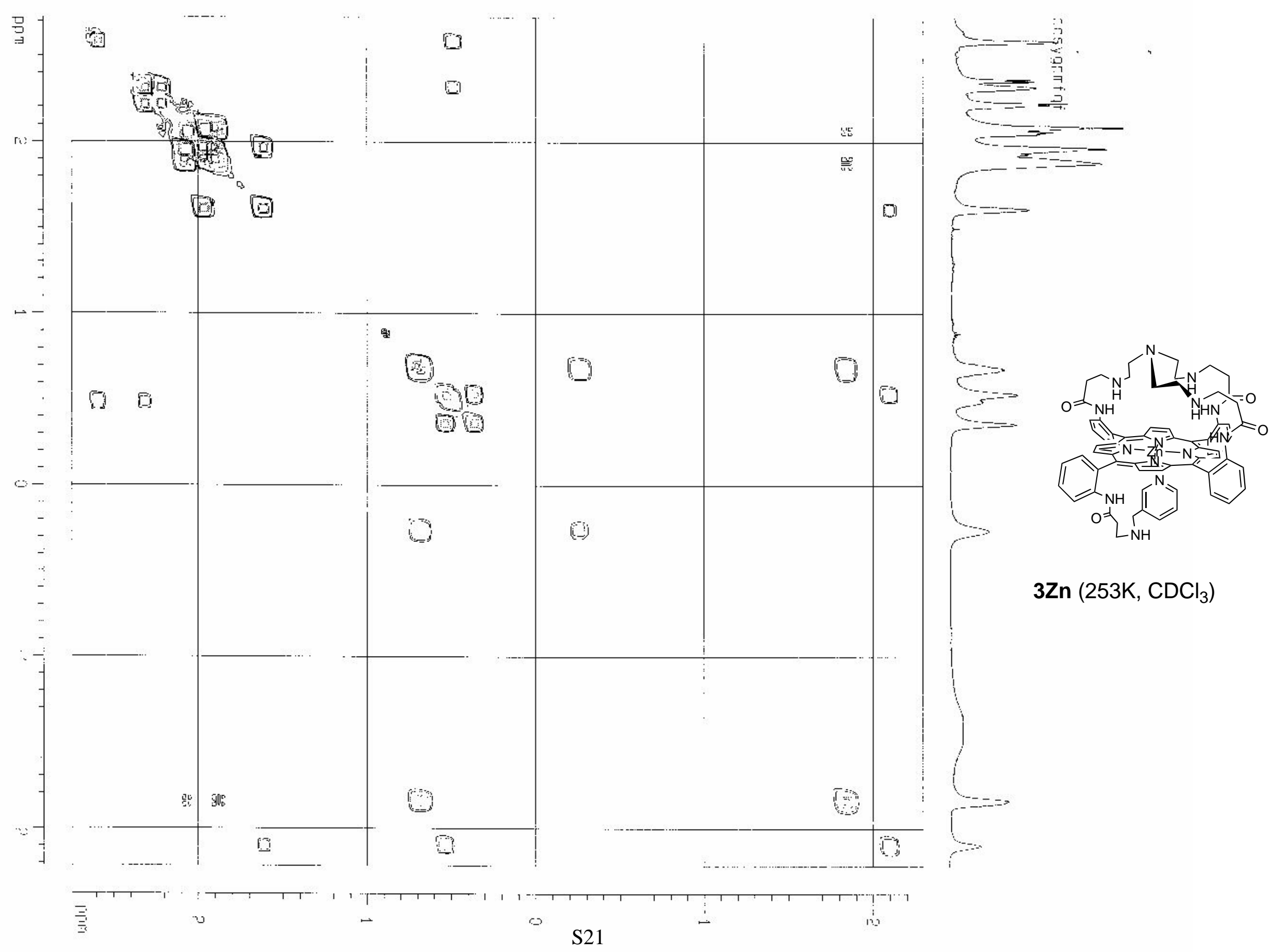




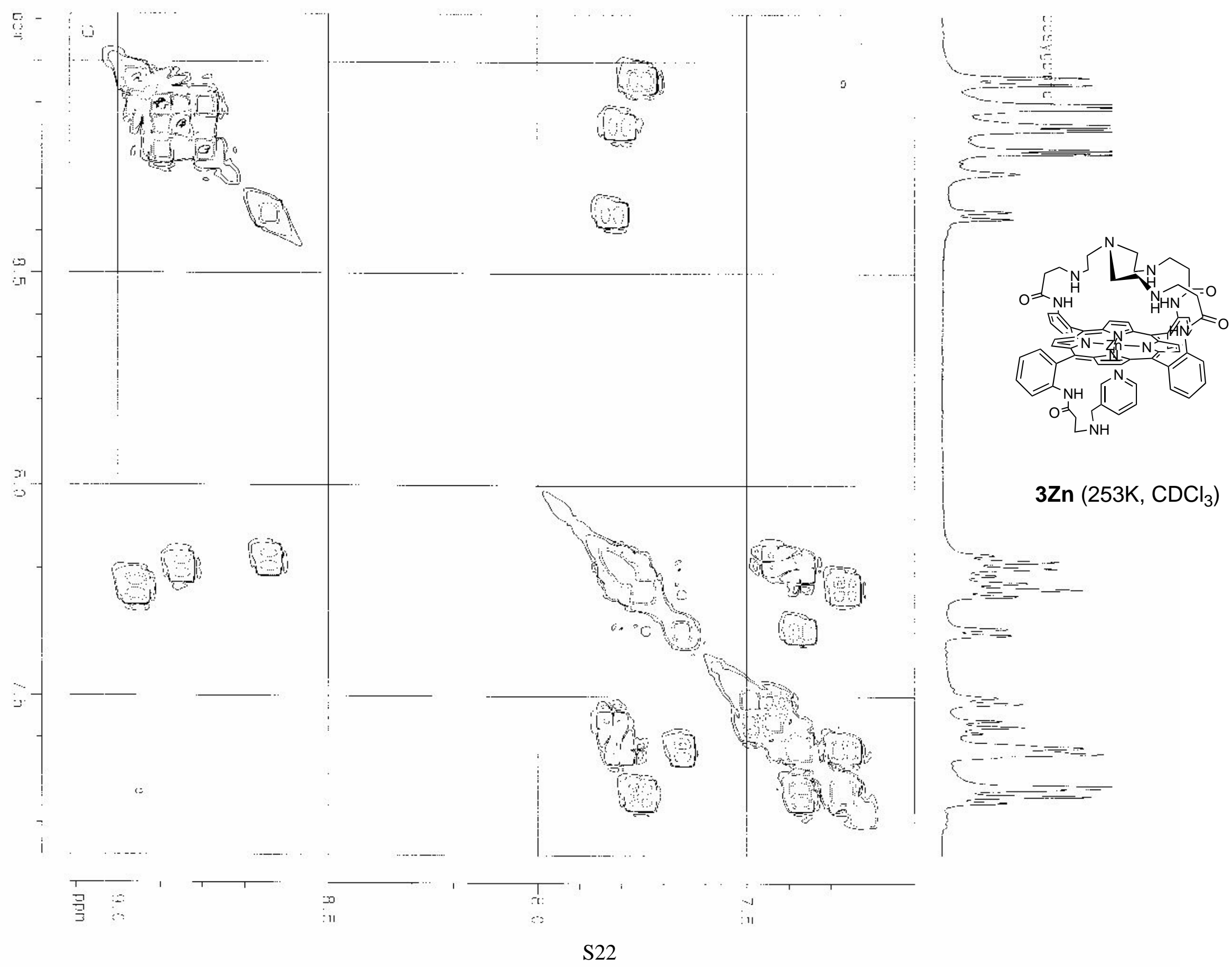



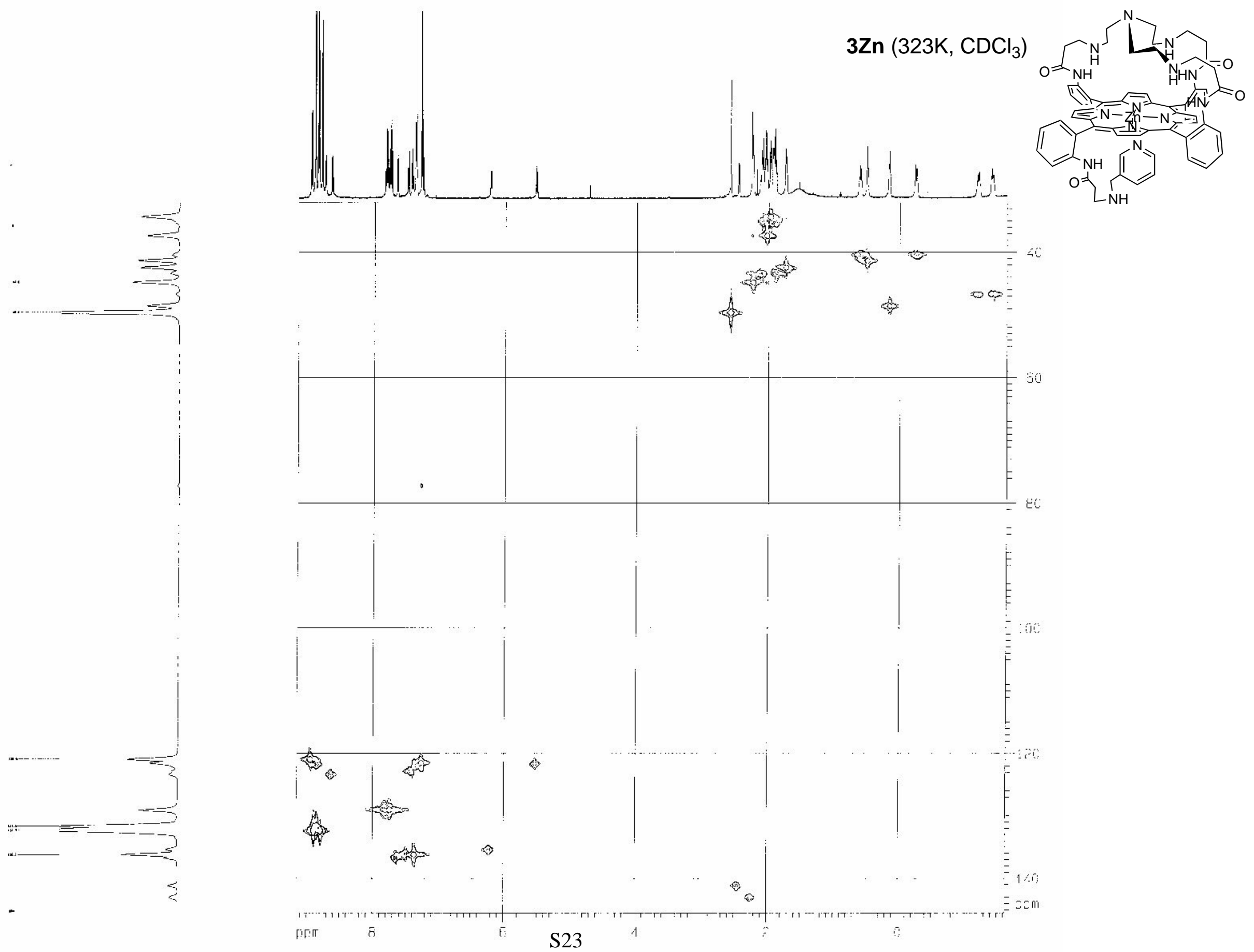


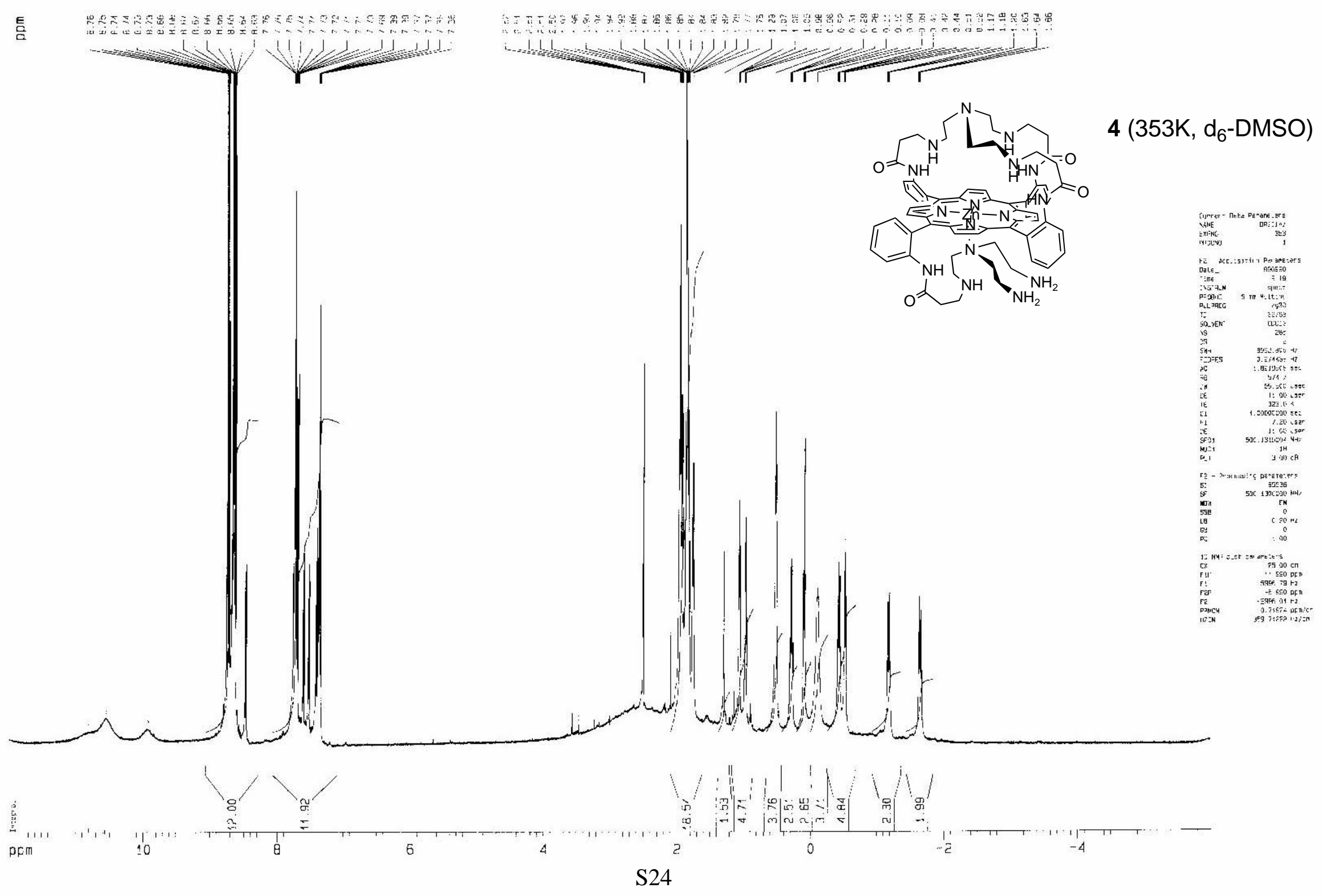




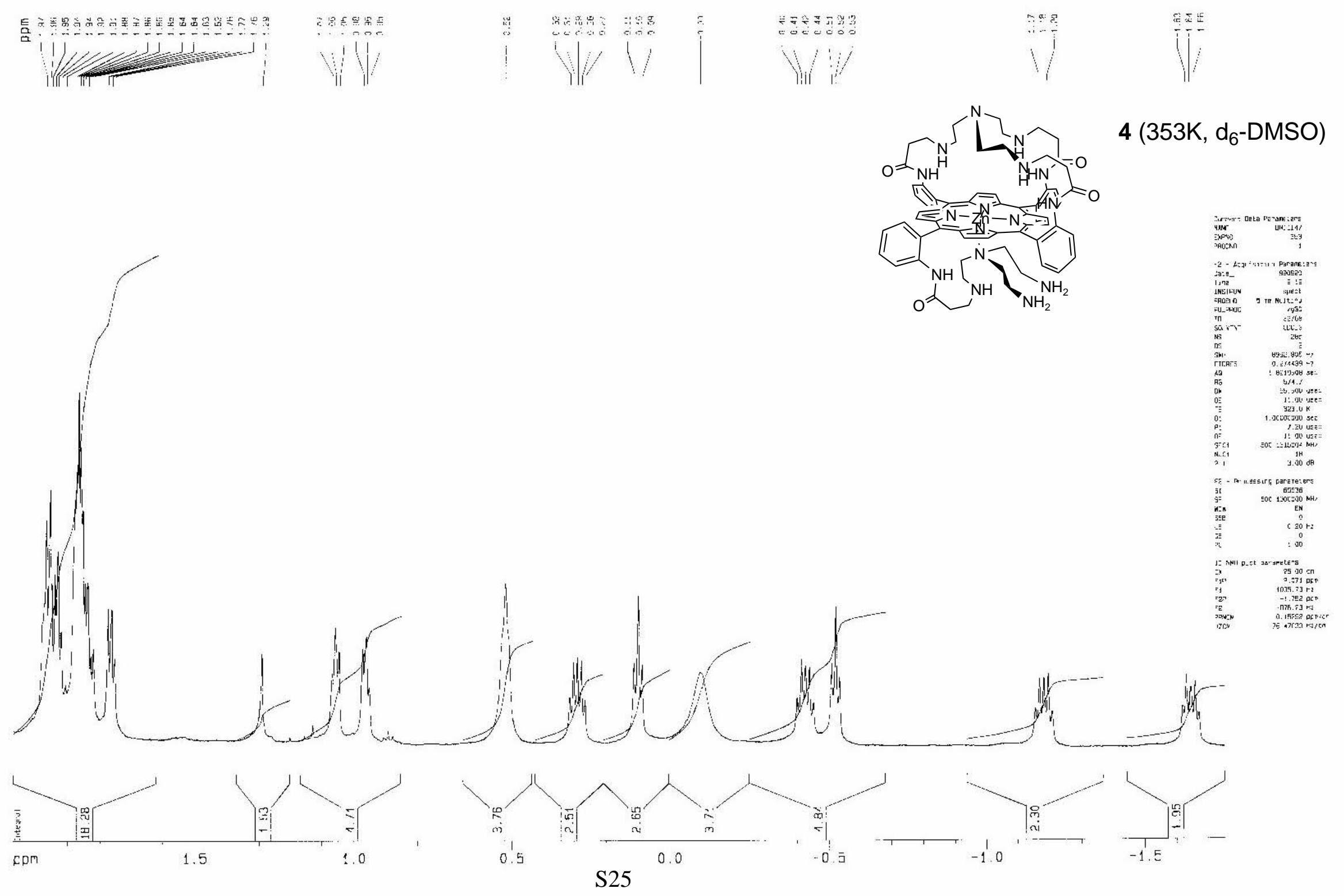




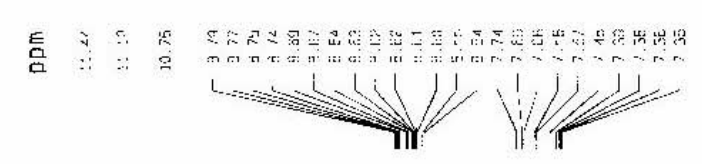

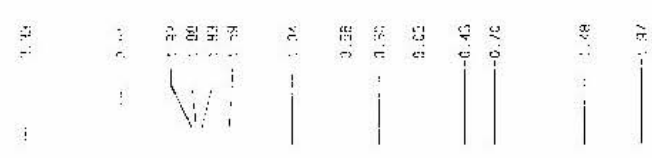

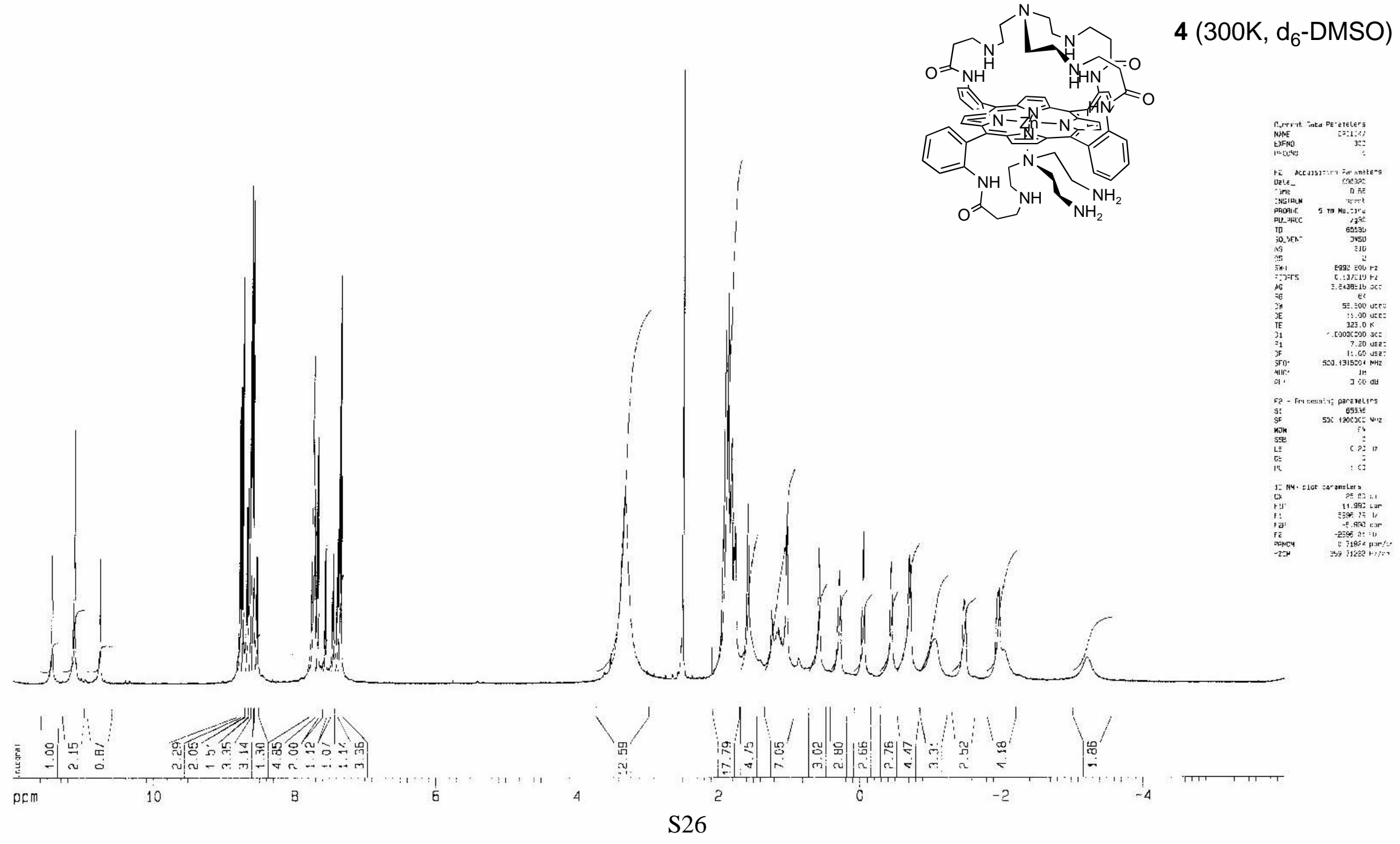




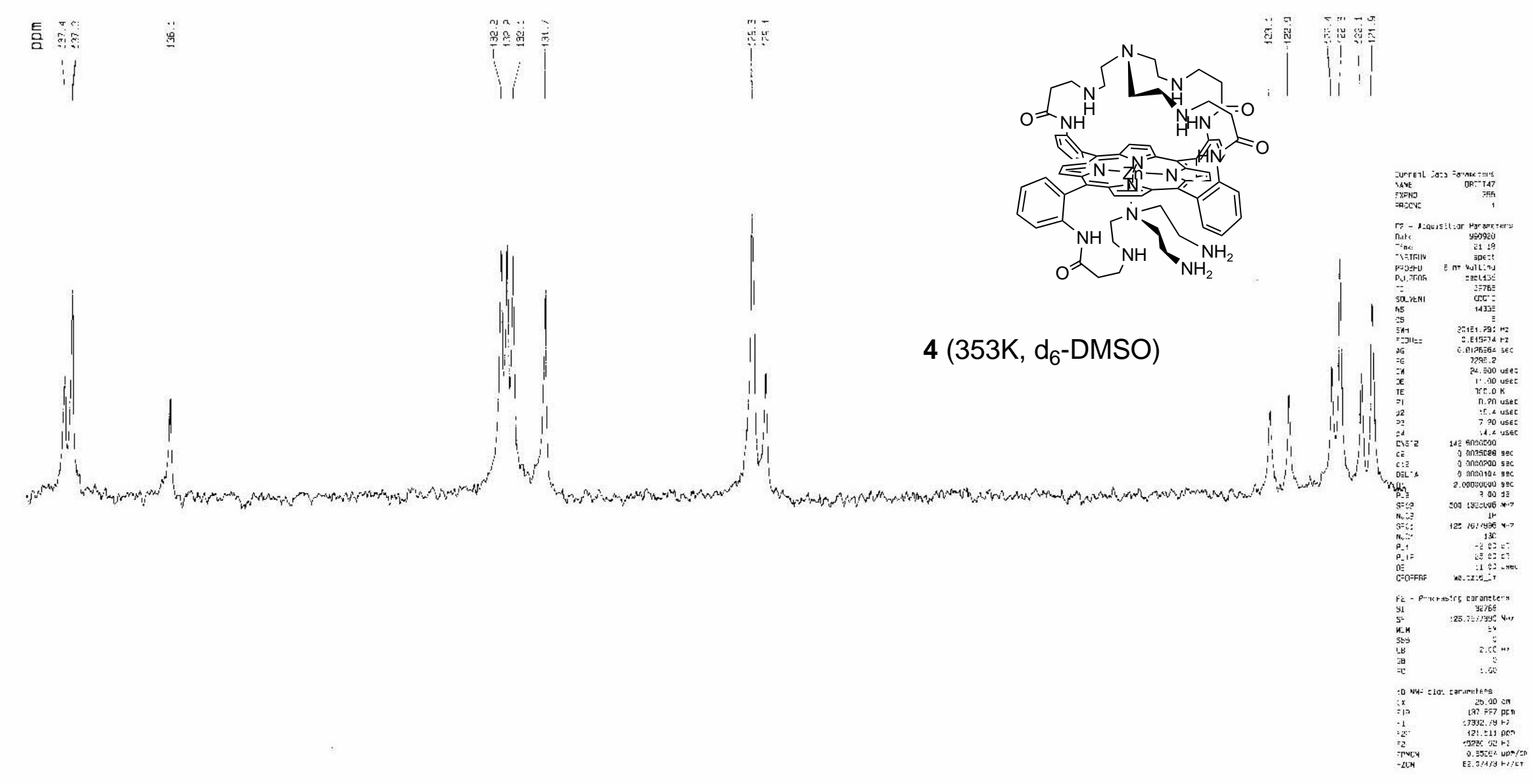




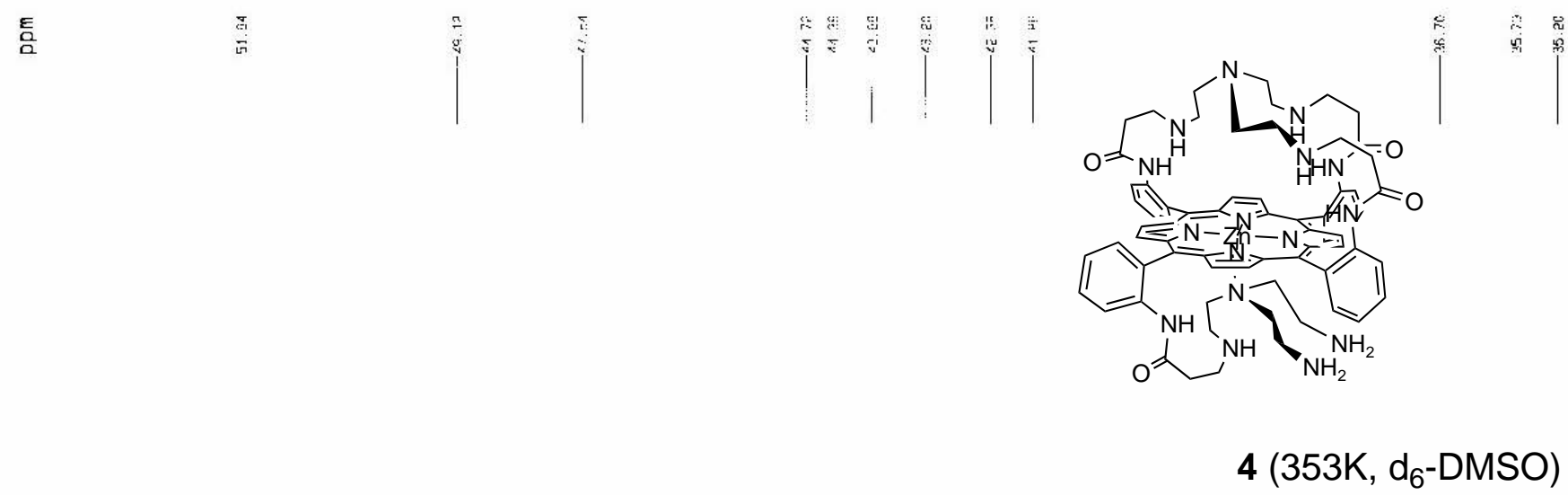

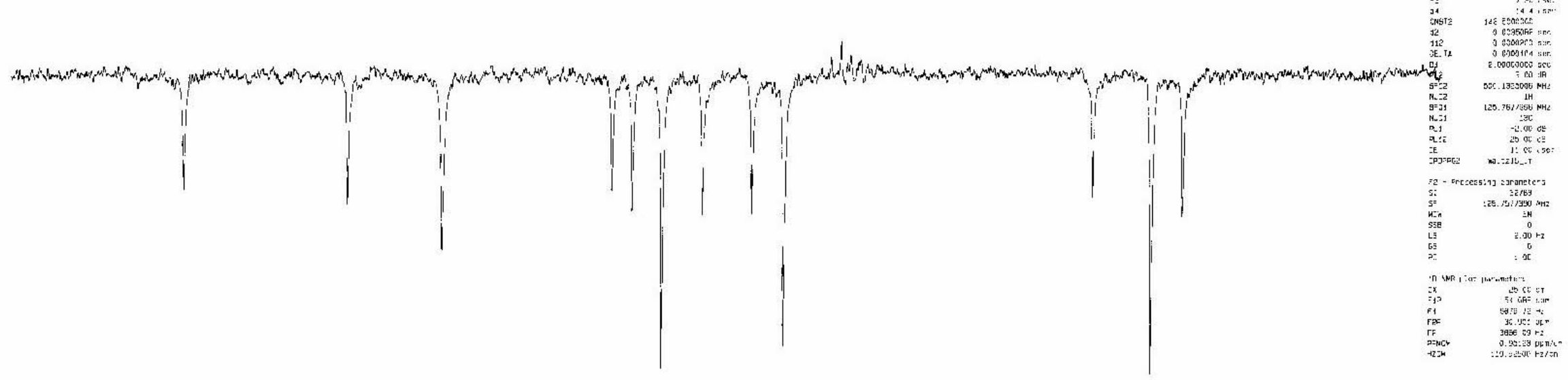




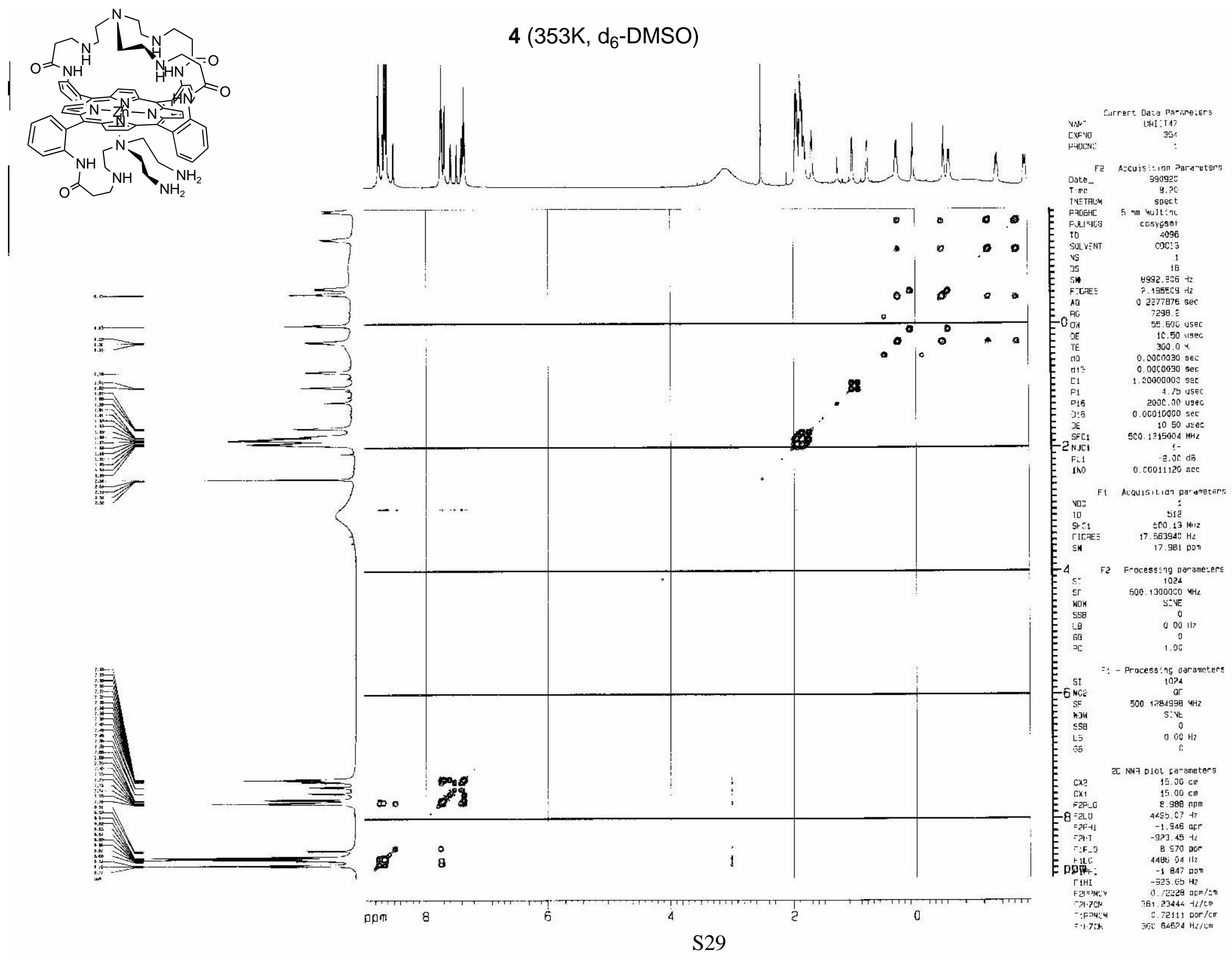




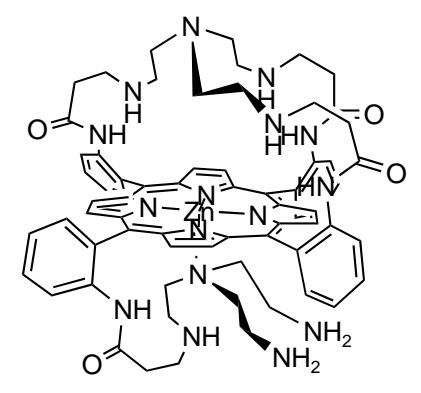

4 (353K, $d_{6}$-DMSO)

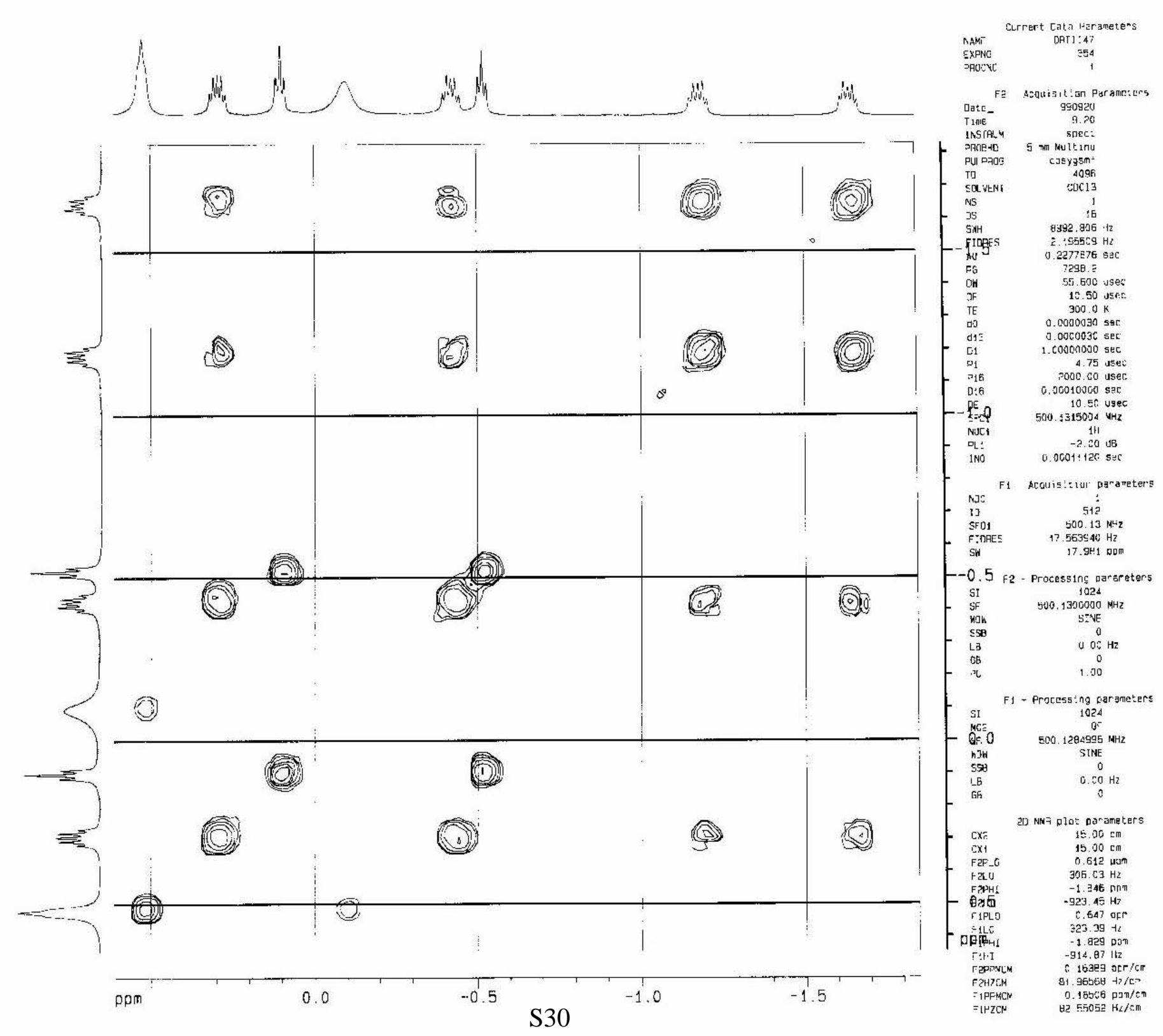

